\title{
Acetylation contributes to hypertrophy- caused maturational delay of cardiac energy metabolism
}

\author{
Arata Fukushima, ${ }^{1,2}$ Liyan Zhang, ${ }^{1}$ Alda Huqi, ${ }^{1}$ Victoria H. Lam, ${ }^{1}$ Sonia Rawat, ${ }^{1}$ Tariq Altamimi, ${ }^{1}$ \\ Cory S. Wagg, ${ }^{1}$ Khushmol K. Dhaliwal, ${ }^{1}$ Lisa K. Hornberger, ${ }^{1}$ Paul F. Kantor, Ivan M. Rebeyka, ${ }^{1}$ \\ and Gary D. Lopaschuk \\ 'Cardiovascular Translational Science Institute, University of Alberta, Edmonton, Alberta, Canada. ${ }^{2}$ Department of \\ Cardiovascular Medicine, Faculty of Medicine and Graduate School of Medicine, Hokkaido University, Sapporo, Japan.
}

\begin{abstract}
A dramatic increase in cardiac fatty acid oxidation occurs following birth. However, cardiac hypertrophy secondary to congenital heart diseases (CHDs) delays this process, thereby decreasing cardiac energetic capacity and function. Cardiac lysine acetylation is involved in modulating fatty acid oxidation. We thus investigated what effect cardiac hypertrophy has on protein acetylation during maturation. Eighty-four right ventricular biopsies were collected from CHD patients and stratified according to age and the absence $(n=44)$ or presence of hypertrophy $(n=40)$. A maturational increase in protein acetylation was evident in nonhypertrophied hearts but not in hypertrophied hearts. The fatty acid $\beta$-oxidation enzymes, long-chain acyl CoA dehydrogenase (LCAD) and $\beta$-hydroxyacyl CoA dehydrogenase ( $\beta H A D)$, were hyperacetylated and their activities positively correlated with their acetylation after birth in nonhypertrophied hearts but not hypertrophied hearts. In line with this, decreased cardiac fatty acid oxidation and reduced acetylation of $L C A D$ and $\beta H A D$ occurred in newborn rabbits subjected to cardiac hypertrophy due to an aortocaval shunt. Silencing the mRNA of general control of amino acid synthesis 5-like protein 1 reduced acetylation of LCAD and $\beta H A D$ as well as fatty acid oxidation rates in cardiomyocytes. Thus, hypertrophy in CHDs prevents the postnatal increase in myocardial acetylation, resulting in a delayed maturation of cardiac fatty acid oxidation.
\end{abstract}

Conflict of interest: The authors have declared that no conflict of interest exists.

Submitted: December 12, 2017

Accepted: April 13, 2018

Published: May 17, 2018

\section{Reference information:}

JCI Insight. 2018;3(10):e99239.

https://doi.org/10.1172/jci.

insight.99239.

\section{Introduction}

Congenital heart disease (CHD) occurs in over $1 \%$ of all live births, with one-third of these infants needing surgery within the first month of life $(1,2)$. Despite a remarkable reduction in operative mortality after corrective surgery, heart failure remains a major determinant of outcomes in patients with corrected CHD $(3,4)$. Multiple factors that predispose to the development of heart failure may include anatomical abnormalities, obligatory ischemic injury during surgery, and cardiac hypertrophy in response to chronic volume and/or pressure overload $(5,6)$. Energy metabolic perturbations during maturation may also play a key role in the development of heart failure associated with CHD (7-9). Immediately after birth, myocardial fatty acid oxidation normally increases rapidly and predominates as a source of myocardial energy production, while glycolytic rates dramatically decline and glucose oxidation rates remain low (10-12). In contrast, cardiac hypertrophy in the neonatal heart results in a more fetal metabolic profile, which includes a greater reliance of the heart on glycolysis in conjunction with the delayed maturation of fatty acid oxidation, thereby compromising the ability of the newborn heart to respond to stress $(8,9)$. This alteration in cardiac energy metabolism is a complex process, which includes alterations in posttranslational control of energy metabolism as well as allosteric and transcriptional regulations of metabolic enzymes (7, 13-16).

Lysine acetylation has been recently identified as a novel posttranslational modification that controls cardiac energy metabolism (16-18). Numerous energy metabolic enzymes can be acetylated, including mitochondrial fatty acid oxidation enzymes (19-21). We previously reported that increased acetylation of fatty acid $\beta$-oxidation enzymes increases the activities of these enzymes and leads to an increase in fatty acid oxidation rates in the newborn hearts as well as hearts from obese mice with heart failure $(16,17,22)$. This mitochondrial acetylation is regulated by the mitochondrial acetyltransferase general control of amino acid 
Table 1. Patient characteristics

\begin{tabular}{|c|c|c|c|c|}
\hline \multirow[b]{2}{*}{ Demographic factors } & \multicolumn{2}{|c|}{ Group 1 (age 21-100 d, $n=38$ ) } & \multicolumn{2}{|c|}{ Group 2 (age 101-200 d, $n=46$ ) } \\
\hline & Nonhypertrophy $(n=23)$ & Hypertrophy $(n=15)$ & Nonhypertrophy $(n=21)$ & Hypertrophy $(n=25)$ \\
\hline Age, d & $50.7 \pm 17.6$ & $66.9 \pm 30.3^{\mathrm{A}}$ & $150 \pm 29.3$ & $152 \pm 28.2$ \\
\hline Body surface area, $\mathrm{m}^{2}$ & $0.23 \pm 0.08$ & $0.23 \pm 0.08$ & $0.28 \pm 0.05$ & $0.28 \pm 0.05$ \\
\hline \multicolumn{5}{|c|}{ Congenital heart diseases } \\
\hline TOF, $n(\%)$ & $7(29 \%)$ & $7(44 \%)$ & $9(43 \%)$ & $13(52 \%)$ \\
\hline DORV, $n(\%)$ & $3(13 \%)$ & $3(19 \%)$ & $0(0 \%)$ & $2(8 \%)$ \\
\hline TGA, $n(\%)$ & 0 (0\%) & $1(6 \%)$ & $3(14 \%)$ & $1(4 \%)$ \\
\hline TA, $n(\%)$ & $1(4 \%)$ & $0(0 \%)$ & $0(0 \%)$ & $1(4 \%)$ \\
\hline $\mathrm{PA}, n(\%)$ & $1(4 \%)$ & $1(6 \%)$ & 0 (0\%) & $2(4 \%)$ \\
\hline BAV, $n(\%)$ & $1(4 \%)$ & $0(0 \%)$ & $0(0 \%)$ & $0(0 \%)$ \\
\hline LVFS, \% & $38 \pm 9.1$ & $37 \pm 7.7$ & $38 \pm 9.6$ & $39 \pm 9.7$ \\
\hline LVML, g/m² & $63 \pm 33$ & $41 \pm 17$ & $64 \pm 44$ & $55 \pm 26$ \\
\hline RV EDD, mm & $1.22 \pm 0.45$ & $1.41 \pm 0.42$ & $1.06 \pm 0.36$ & $1.11 \pm 0.26$ \\
\hline RVAW, mm & $0.38 \pm 0.04$ & $0.43 \pm 0.07$ & $0.32 \pm 0.04$ & $0.51 \pm 0.1$ \\
\hline RVAW (Z-score) & $0.84 \pm 0.75$ & $3.26 \pm 1.19^{A}$ & $0.97 \pm 0.72$ & $4.37 \pm 1.71^{A}$ \\
\hline TAPSE & $1.13 \pm 0.27$ & $0.81 \pm 0.1$ & $1.18 \pm 0.22$ & $0.98 \pm 0.4$ \\
\hline \multicolumn{5}{|c|}{$\begin{array}{l}\text { Data represent mean } \pm \mathrm{SD} \text {. }{ }^{A} P<0.05, t \text { test. TOF, tetralogy of Fallot; VSD, ventricular septal defect; AVSD, atrioventricular septal defect; DORV, double } \\
\text { outlet right ventricle; TCA, transposition of the great arteries; TA, tricuspid atresia; PA, pulmonary atresia; BAV, bicuspid aortic valve; LV, left ventricular; } \\
\text { EDD, end-diastolic diameter; LVFS, LV fractional shortening; LVMI, LV mass index; RV, right ventricular; RVAWT, RV anterior wall thickness; TAPSE, } \\
\text { tricuspid annular plane systolic excursion. }\end{array}$} \\
\hline
\end{tabular}

synthesis 5-like (GCN5-like) protein 1 (GCN5L1) (23), while deacetylation is regulated by mitochondrial deacetylases such as sirtuin 3 (SIRT3) (19). However, the effect cardiac hypertrophy has on acetylation control of energy metabolism in the newborn heart has been unknown.

In this study, we determined whether the presence of cardiac hypertrophy alters acetylation control of fatty acid oxidation along with regulatory enzymes of glycolysis and glucose oxidation in human neonatal hearts, using right ventricular (RV) biopsy samples from CHD patients. To examine actual metabolic rates, neonatal rabbits with volume overload hypertrophy were also used as an animal model of CHD $(8,9)$. Furthermore, we determined the effects of Gcn5l1 gene knockdown on developmental alterations in acetylation and energy metabolism in hypertrophied $\mathrm{H} 9 \mathrm{c} 2$ cardiomyocytes.

\section{Results}

Myocardial characteristics of the study subjects. Demographic and baseline characteristics of the studied patients are shown in Table 1. Among the 84 samples, 38 were in age group 1 (21-100 days), and 46 were in age group 2 (101-200 days). Of the 84 samples, 40 samples were identified as hypertrophied hearts, based on the echocardiographically assessed Z-score of more than 2.0. Similar numbers of hypertrophic and nonhypertrophic myocardial samples were obtained for each age group. Echocardiographic study revealed that there was no difference in preprocedural left ventricular (LV) function among the 2 age groups, regardless of the presence or absence of hypertrophy. RV end-diastolic diameter and tricuspid annular plane systolic excursion, an index of RV systolic function, were not different among groups. RV anterior wall thickness (RVAW) was greater in hypertrophied hearts than nonhypertrophied hearts in age group 1, and Z-scores of RVAW were significantly increased in hypertrophied hearts compared with nonhypertrophied hearts in each age group.

Myocardial protein lysine acetylation and its regulatory enzymes in congenital diseased hearts. To investigate the effect of age and cardiac hypertrophy on cardiac lysine acetylation, we first examined the total protein acetylation in nonhypertrophied and hypertrophied hearts (Figure 1). The level of cardiac pro- 
A

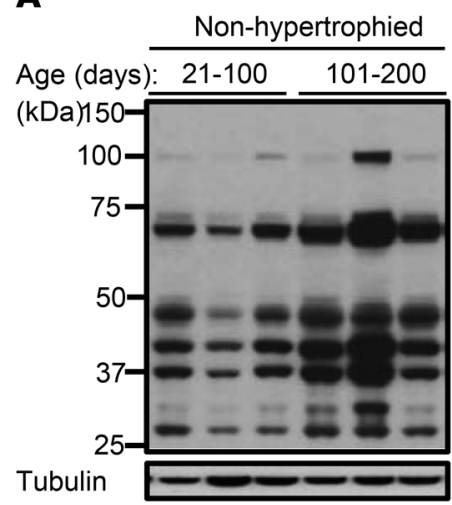

Age (days): 21-100 101-200

SIRT1

SIRT2

SIRT3

SIRT4

SIRT5

SIRT6

GCN5

GCN5L1

Tubulin
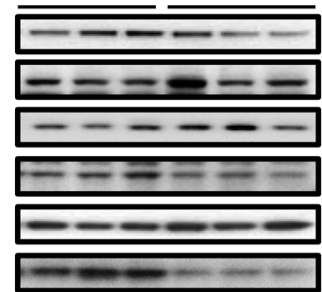

C Non-hypertrophied
Hypertrophied

\begin{tabular}{c} 
Hypertrophied \\
\hline $21-100 \quad 101-200$ \\
\hline
\end{tabular}

B
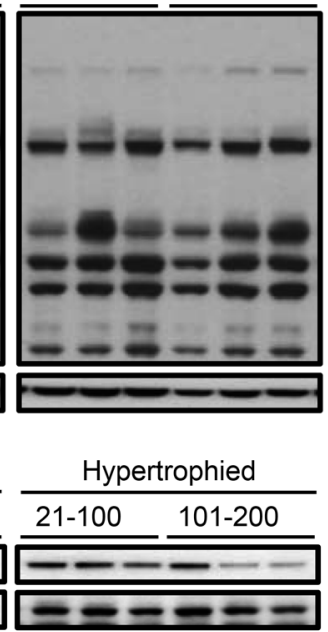

D

$\mathbf{F}$
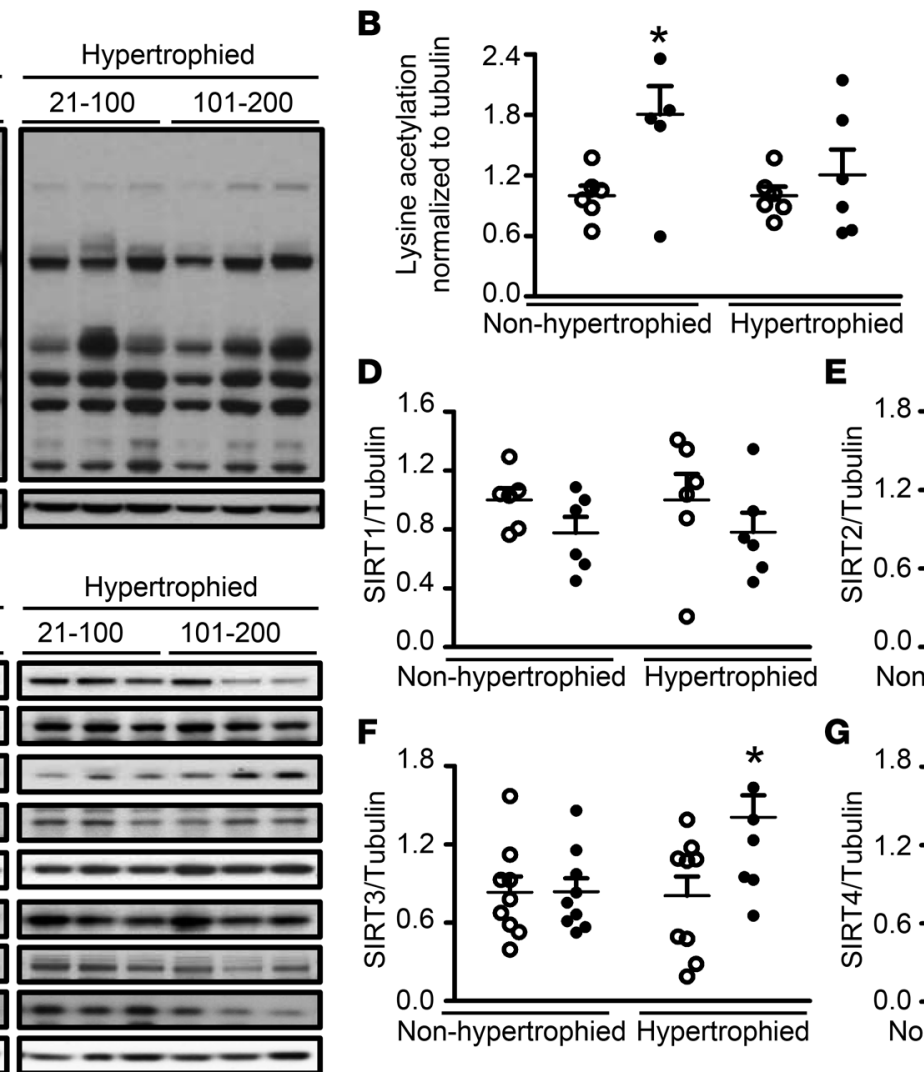

Non-hypertrophied $\overline{\text { Hypertrophied }}$

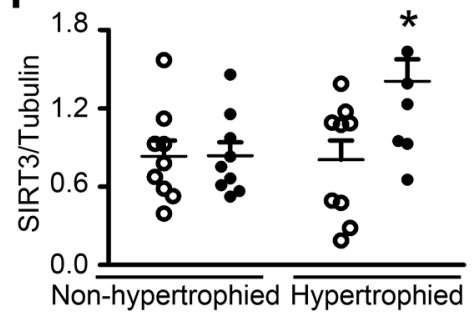

E

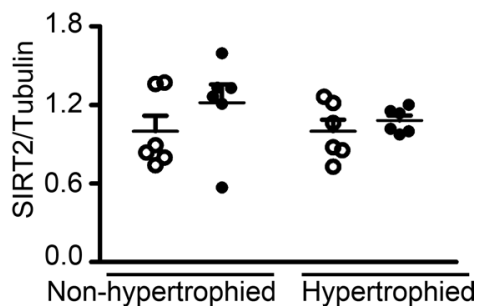

G

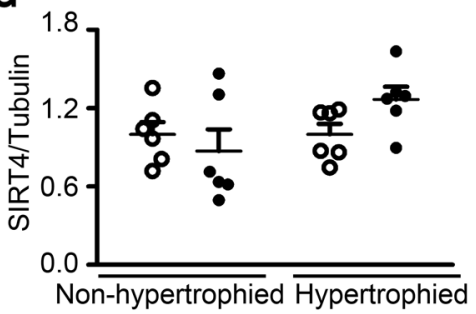

$\mathbf{K}$

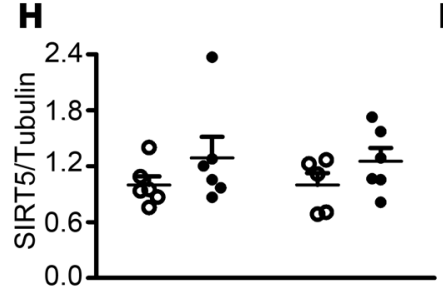

I

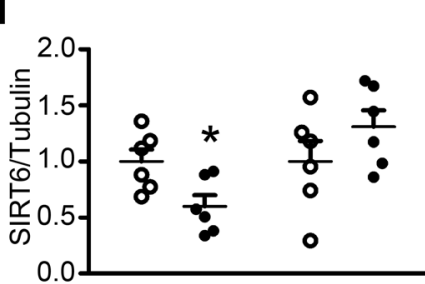

J

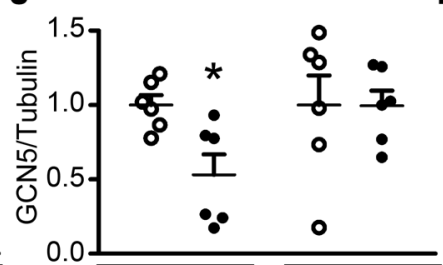

- Age (days): 101-200
- Age (days): $21-100$

Non-hypertrophied $\overline{\text { Hypertrophied Non-hypertrophied }} \overline{\text { Hypertrophied }}$ Non-hypertrophied $\overline{\text { Hypertrophied Non-hypertrophied Hypertrophied }}$

Figure 1. Overall acetylation of cardiac protein in neonatal human hearts, and postnatal alterations in sirtuins and acetyltransferases. (A and B) Representative immunoblots of total protein acetylation and its densitometric analysis normalized to tubulin ( $n=6 /$ group). (C-I) Representative immunoblots of sirtuin 1-6 (SIRT1-6) and their densitometric analysis normalized to tubulin ( $n=6$ /group, C-E and G; $n=8 /$ group, F), (C, J and K) Representative immunoblots of nuclear acetyltransferase general control of amino acid synthesis 5 (GCN5) ( $n=6 /$ group, J), mitochondrial acetyltransferase GCN5-like protein 1 (GCN5L1, $n=9$ /group, K) and their densitometric analysis normalized to tubulin. All lanes were run on the same gel but were noncontiguous, except for SIRT2, SIRT6, GCN5, and GCN5L1, which were from different gels for each group (hypertrophy/nonhypertrophied). Values represent mean \pm SEM. * $P<0.05,2$-way ANOVA, comparing ages 21-100 days and 101-200 days in the same group. Black circles denote age of 21-100 days, and white circles denote age of 101-200 days.

tein acetylation was age-dependently elevated in nonhypertrophied samples, whereas the increase was diminished in hypertrophied hearts (Figure 1, A and B). To understand what may be responsible for these differences in acetylation, we measured the expression of a diverse family of acetyltransferase and deacetylase enzymes (Figure 1C). Protein expression of cardiac SIRT1 and SIRT2 were unaltered among groups, regardless of the presence or absence of hypertrophy (Figure 1, C-E). In contrast, the mitochondrial deacetylase SIRT3 was unchanged in nonhypertrophied hearts, but age-dependently upregulated in hypertrophied hearts, while other mitochondrial deacetylases, SIRT4 and SIRT5, remained unaltered among age groups. The expression of nuclear deacetylase SIRT6 and the acetyltransferase GCN5 decreased in an age-dependent manner in nonhypertrophied hearts, whereas this decrease was attenuated in hypertrophied hearts. Of importance, the mitochondrial acetyltransferase GCN5L1 increased following aging in nonhypertrophied hearts, but the increase was markedly declined in hypertrophied hearts (Figure 1, C and K). The increase in GCN5L1 was consistent with the overall increase in protein 
acetylation following aging in nonhypertrophied hearts, with the decrease in GCN5L1 being consistent with the decreased acetylation with aging in the hypertrophied hearts (Figure 1, A and B).

Acetylation control of fatty acid $\beta$-oxidation enzymes in congenital diseased hearts. We previously demonstrated in rabbits and pigs that cardiac hypertrophy delays the normal maturational increase in fatty acid oxidation $(8,9)$, and acetylation of fatty acid $\beta$-oxidation enzymes positively regulates their activities and fatty acid oxidation rates (16). To understand if this mechanism also occurs during maturation of human hearts, we examined the levels of acetylation of the fatty acid $\beta$-oxidation enzymes long-chain acyl CoA dehydrogenase $(\mathrm{LCAD})$ and $\beta$-hydroxyacyl CoA dehydrogenase $(\beta \mathrm{HAD})$ in hypertrophied and nonhypertrophied human hearts. Despite unaltered total protein expression of cardiac LCAD and $\beta \mathrm{HAD}$ (Figure $2, \mathrm{~A}-\mathrm{C}$ ), the acetylation levels of LCAD and $\beta \mathrm{HAD}$ were significantly increased following aging in nonhypertrophied hearts (Figure 2, D-F). However, this maturational increase in LCAD and $\beta H A D$ was not seen in hypertrophied hearts (Figure 2, D-F). The increased acetylation of LCAD and $\beta \mathrm{HAD}$ was mirrored by increased activity of $\mathrm{LCAD}$ and $\beta \mathrm{HAD}$ in nonhypertrophied hearts (Figure 2, $\mathrm{G}$ and $\mathrm{H}$ ). A positive correlation between acetylated levels of LCAD or $\beta \mathrm{HAD}$ and the activity of LCAD or $\beta \mathrm{HAD}\left(\mathrm{R}^{2}=0.49\right.$ and 0.54 , respectively, $n=6 /$ group, $P<0.01$ ) was evident in nonhypertrophied hearts (Figure 2, I and J). However, age-dependent increases in LCAD and $\beta \mathrm{HAD}$ activity were not seen in hypertrophied hearts (Figure 2, G and $\mathrm{H}$ ), nor was a correlation seen between LCAD and $\beta \mathrm{HAD}$ activity and acetylated LCAD and $\beta \mathrm{HAD}$, respectively (Figure 2, I and J), suggesting that cardiac hypertrophy decreased the maturational increase in fatty acid oxidation in human hearts in part through a mechanism involving decreased acetylation of fatty acid $\beta$-oxidation enzymes.

Increased acetylation of peroxisome proliferator-activated receptor $\gamma$ coactivator $1 \alpha$ in hypertrophied newborn hearts. Mitochondrial biogenesis increases following birth, due in part to an increase in peroxisome proliferator-activated receptor $\gamma$ coactivator $1 \alpha(\mathrm{PGC} 1 \alpha)$ transcriptional activity (24). Following birth, an agedependent increase in PGC1 $\alpha$ expression was seen, which was not altered by the presence of hypertrophy (Figure 3, A and C). Recent studies have shown that acetylation of PGC1 $\alpha$ activity can reduce its activity (25). Of interest is that a maturational increase in acetylation of PGC1 $\alpha$ was observed in the hypertrophied hearts (Figure 3, B and D), despite a decrease in overall acetylation in these hearts (Figure 1, A and B). This was accompanied by maturational increases in protein levels and activity of citrate synthase (CS) in nonhypertrophied hearts (Figure 3, E-G), but not in hypertrophied hearts, consistent with a compromised mitochondrial biogenesis in hypertrophied hearts. This lack of increase in CS with age in hypertrophied hearts is consistent with an acetylation and inhibition of PGC1 $\alpha$ in the hypertrophied hearts.

Cardiac hypertrophy enhances enzymes related to triacylglyceride and ceramide synthesis. To understand whether decreased mitochondrial biosynthesis and fatty acid oxidation in hypertrophied hearts could shift intracellular lipids toward biosynthesis of triacylglyceride and ceramide in CHDs hearts, we examined protein expression of key enzymes involved in triacylglyceride and ceramide synthesis. Protein expression of diacylglycerol acyltransferase 2 (DGAT2), a major enzyme catalyzing triacylglyceride biosynthesis from diacylglycerol, was enhanced with age only in hypertrophied hearts (Figure $3 \mathrm{H}$ ), whereas the expression of adipose triglyceride lipase (ATGL), a major triacylglyceride lipase in the heart, was unaltered following aging in both hypertrophied and nonhypertrophied hearts (Figure 3I). In addition, protein levels of serine palmitoyltransferase 1 (SPT1) and SPT2, the rate-limiting enzymes for de novo ceramide synthesis, were both elevated with age only in hypertrophied hearts but not in nonhypertrophied hearts (Figure 3, J and K).

Acetylation control of fatty acid $\beta$-oxidation enzymes in hypertrophied neonatal rabbit hearts. To further investigate the effects of hypertrophy on acetylation control of cardiac energy metabolism in the newborn, we directly assessed energy metabolism in neonatal rabbit hearts subjected to a volume overload-induced hypertrophy. Cardiac hypertrophy was produced by creating an aortocaval shunt in 7-day-old rabbits, as described previously $(8,9)$. By 21 days of age, a 33\% increase in cardiac mass was observed (8), and its myocardial metabolic profile is thought to reflect that in age group 2 (101-200 days) in humans (26).

Subsequent biventricular working heart perfusions of these hearts showed a prominent depression (by $58 \%$ ) in palmitate oxidation rates in hypertrophied hearts compared with hearts from sham-operated rabbits (Figure 4A). In contrast, glycolytic rates were 293\% higher in hypertrophied hearts compared with nonhypertrophied hearts (Figure 4B). These data confirmed the delayed maturation of fatty acid oxidation seen in hypertrophied newborn hearts (7-9). Glucose oxidation rates were similar in these two groups (Figure 4C). As a result, overall myocardial ATP production rates were decreased in hypertrophied hearts, which was mainly attributed to a decrease by $29 \%$ in ATP production rates from palmitate oxidation. This deprivation of ATP production in hypertrophied hearts was accompanied by their lower level of functional work compared 
A

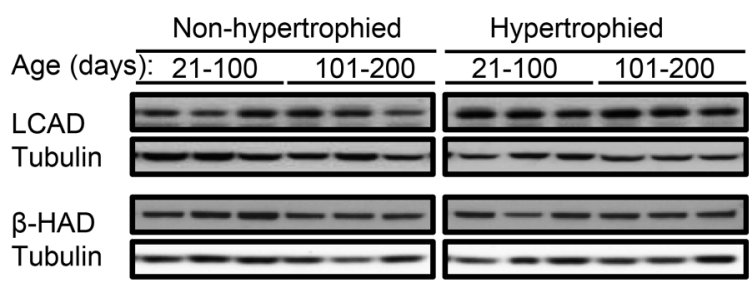

D

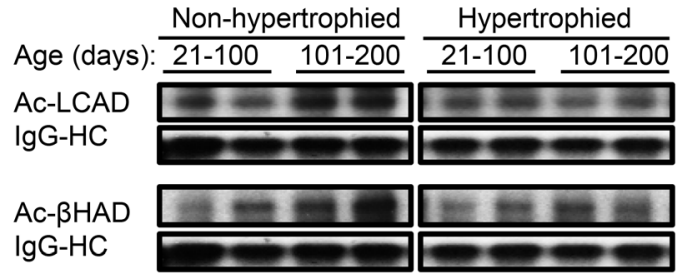

B

- Age (days): $21-100$

- Age (days): 101-200

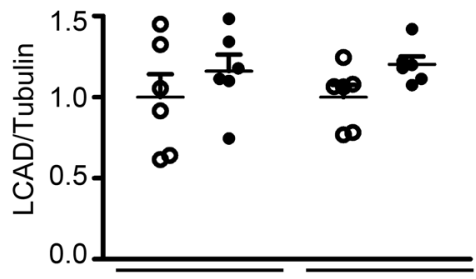

Non-hypertrophied $\overline{\text { Hypertrophied }}$

E

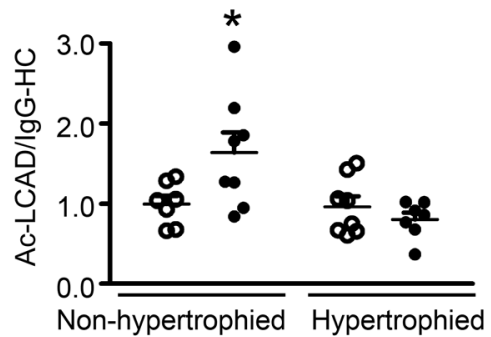

C

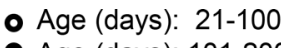

- Age (days): $101-200$

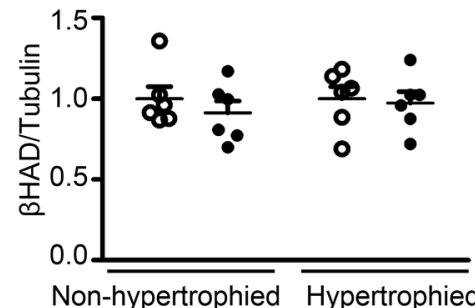

$\mathbf{F}$

- Age (days): 21-100

- Age (days): 101-200

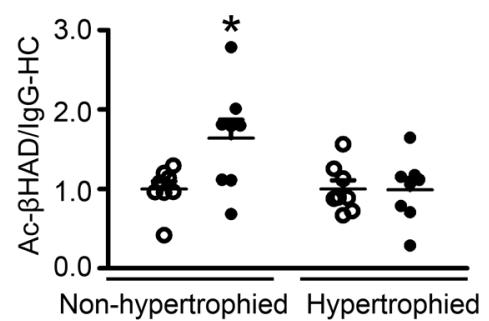

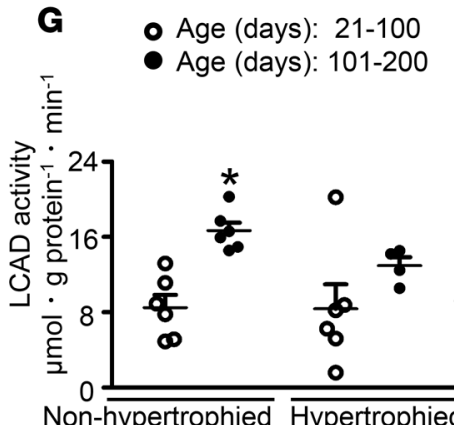

Non-hypertrophied Hypertrophied
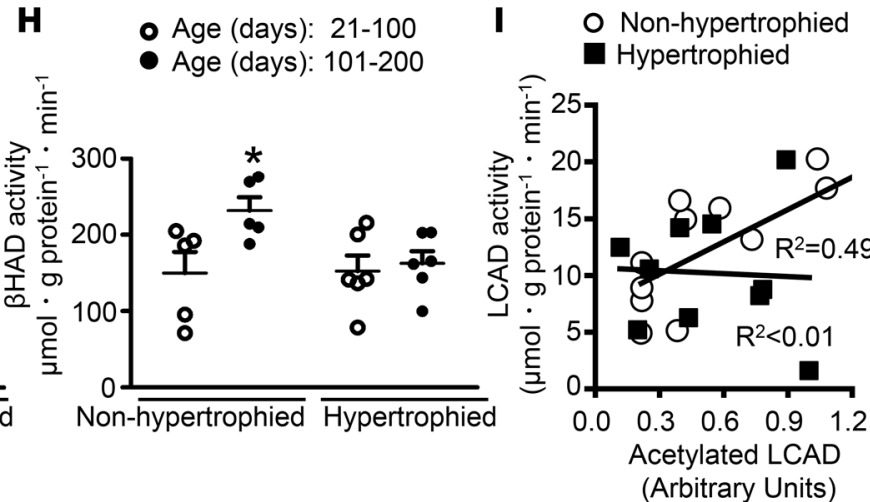

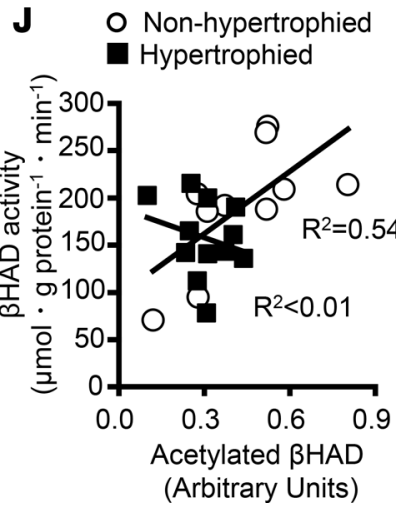

Figure 2. Acetylation control of fatty acid $\beta$-oxidation enzymes in neonatal human hearts. (A-C) Representative immunoblots and densitometric analysis of long-chain acyl CoA dehydrogenase (LCAD, $n=6$ /group) and $\beta$-hydroxyacyl CoA dehydrogenase ( $\beta H A D, n=6 /$ group). (D-F) Representative immunoblots and densitometric analysis of acetylated LCAD (AC-LCAD, $n=6 /$ group) and acetylated $\beta$ HAD (Ac- $\beta$ HAD, $n=6 /$ group). (G and H) LCAD activity ( $n=6$ / group) and $\beta \mathrm{HAD}$ activity ( $n=6$ /group). (I and J) Correlation between activity and acetylation for LCAD ( $n=6 /$ group) and $\beta H A D$ ( $n=6 /$ group), respectively. Lanes were run on the same gel but were noncontiguous. Values represent mean $\pm \mathrm{SEM}$. ${ }^{*} P<0.05,2$-way ANOVA and linear regression analysis using the least squares methods, comparing ages 21-100 days and 101-200 days in the same group. $\mathrm{R}^{2}$, correlation coefficient; IgG-HC, antibody lgG heavy chain.

with sham hearts $\left(12.9 \pm 0.79\right.$ vs. $\left.19.9 \pm 1.40 \mathrm{mmHg} \times \min ^{-1} \times 10^{3}, n=7, P<0.05\right)$, while heart rates were similar between hypertrophied and sham hearts during aerobic perfusion ( $236 \pm 11$ vs. $247 \pm 7 \mathrm{bpm} / \mathrm{min}, n=$ $7, P=\mathrm{NS}$ ). Although protein expression of LCAD and $\beta \mathrm{HAD}$ was unaltered by hypertrophy (Figure $4, \mathrm{D}-\mathrm{F}$ ), their acetylation levels were significantly lower in hypertrophied hearts than sham hearts (Figure 4, G-I), as were their activities (Figure 5, A and B). This paralleled what was observed in hypertrophied human newborn hearts in age group 2 (101-200 days), as shown in Figure 2, E-H, because this age of human hearts corresponds to 21-day-old rabbit hearts. A positive correlation between acetylated levels of LCAD or of $\beta \mathrm{HAD}$ and their activities was also evident (Figure 5, C and D).

Of interest, the decrease in acetylation of LCAD and $\beta$ HAD was accompanied by a repressed GCN5L1 protein expression (Figure 5, E and F), while SIRT3 expression was unaltered (Figure 5, E and G). Importantly, a positive correlation between acetylated levels of LCAD or of $\beta \mathrm{HAD}$ and palmitate oxidation rates (Figure 5, H and I) was evident, suggesting that decreased acetylation of fatty acid $\beta$-oxidation enzymes contributes to a decrease in fatty acid oxidation and compromised cardiac energetics in neonatal hearts with volume-overloaded hypertrophy. 


A
Age (days $\frac{\text { Non-hypertrophied }}{21-100 \quad 101-200}$ Hypertrophied
PGC1a
Tubulin
B
Age (days): 21 Non-hypertrophied
Ac-PGC10
IgG-LC

E
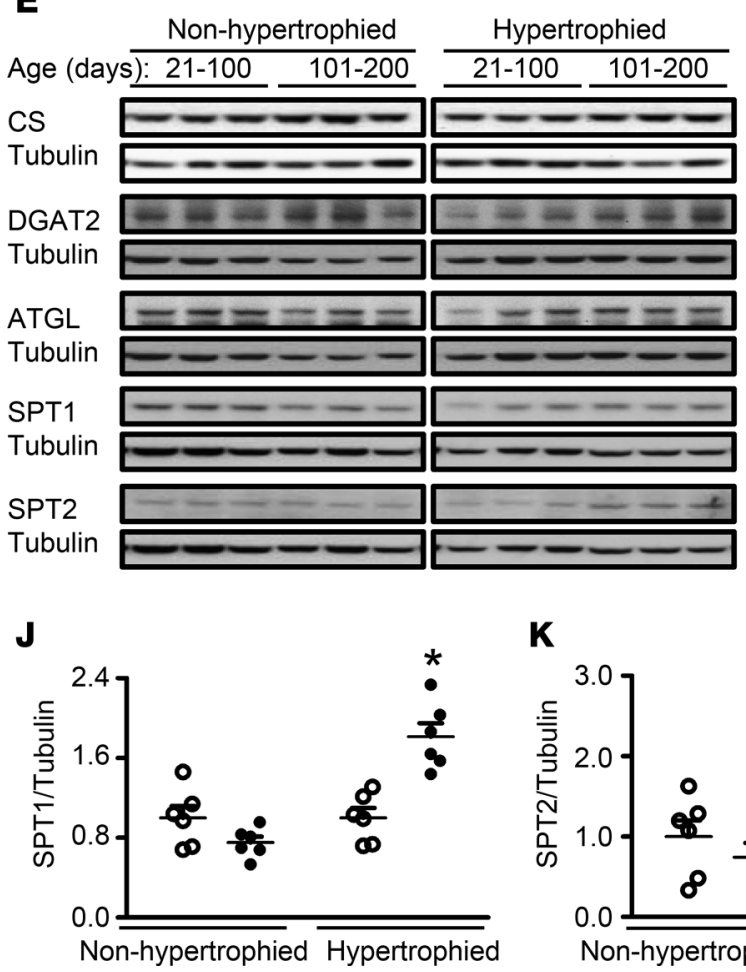

C

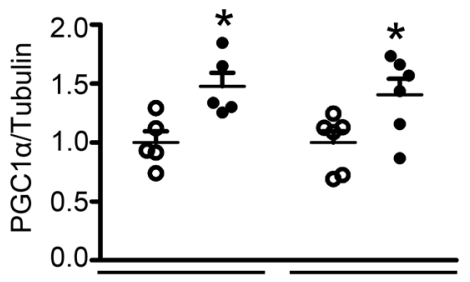

F

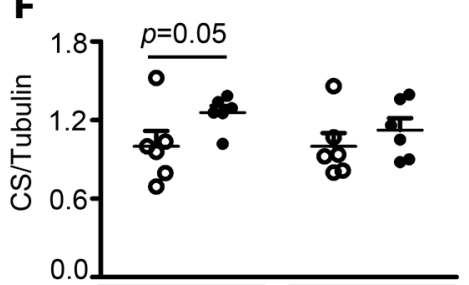

Non-hypertrophied $\overline{\text { Hypertrophied }}$

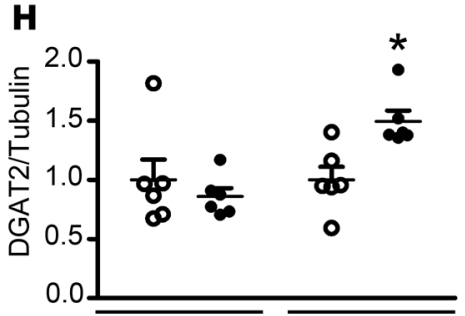

Non-hypertrophied Hypertrophied

$\mathbf{K}$

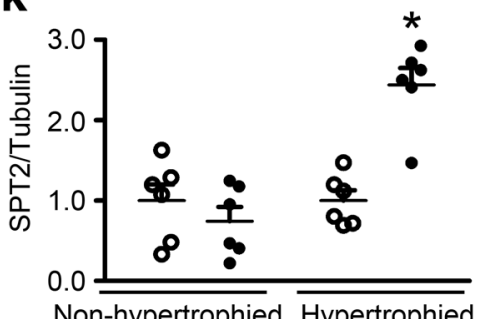

D

- Age (days): $21-100$

- Age (days): 101-200

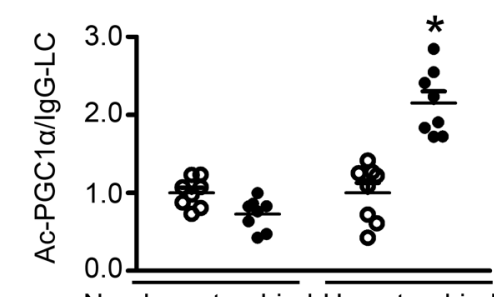

Non-hypertrophied Hypertrophied

G

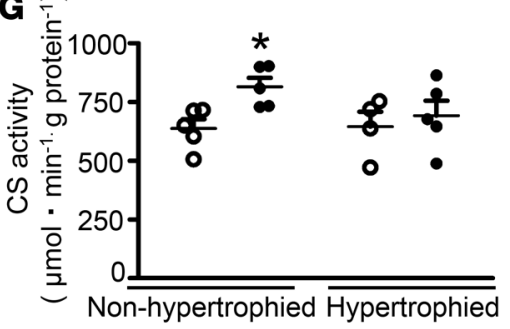

I

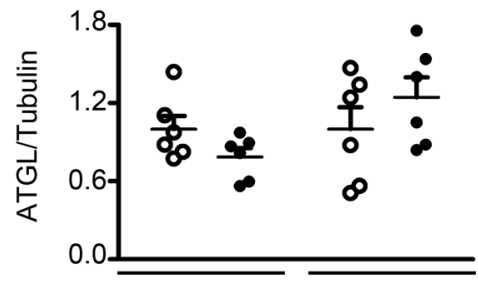

Non-hypertrophied Hypertrophied

Figure 3. Acetylation of peroxisome proliferator-activated receptor $\gamma$ coactivator $1 \alpha$, and expression of enzymes for triacylglycerol/ceramide synthesis in human neonatal hearts. (A-D and F) Representative immunoblots and analysis of peroxisome proliferator-activated receptor $\gamma$ coactivator $1 \alpha(P G C 1 \alpha)$ ( $n=6$ 6/group), acetylated PGC1 $\alpha$ (Ac-PGC1 $\alpha, n=8$ /group), and citrate synthase (CS, $n=6 /$ group). (C) CS activity ( $n=5 /$ group). (E and $\mathbf{H - K}$ ) Representative immunoblots and analysis of diacylglycerol acyltransferase 2 (DCAT2), adipose triglyceride lipase (ATCL), and serine palmitoyltransferase 1 and 2 (SPT1 or SPT2) ( $n=6$ /group in H-K). All lanes were run on the same gel but were noncontiguous, except for ATCL and SPT1, which were from different gels for each group (hypertrophy/nonhypertrophied). Values represent mean \pm SEM. ${ }^{*} P<0.05$, 2-way ANOVA, comparing ages $21-100$ days and $101-200$ days in the same group. IgG-LC, antibody IgG light chain; PC, positive control from the heart lysate without incubation of antibody. White circles denote age of 21-100 days, and black circles denote age of 101-200 days.

Cardiac hypertrophy enhances glycolysis via upregulation of pyruvate dehydrogenase kinase 4 in hypertrophied human newborn hearts. The lower fatty acid oxidation rates observed in hypertrophied newborn rabbit hearts were accompanied by a greater reliance on glycolysis (Figure 4B), metabolic effects that parallel what has been previously reported in RV hypertrophied hearts from adult rats secondary to pulmonary hypertension (27). Since we could not measure glycolysis in the human heart samples, we examined what transcriptional factors and enzymes might be involved in the effects of hypertrophy on the maturational changes in glucose metabolism. Protein expression of HIF1 $\alpha$, a key transcriptional factor involved in the upregulation of glycolysis, was only increased in hypertrophied human hearts with age but was not increased in nonhypertrophied hearts (Figure 6 , A and B). Similar, phosphorylation of pyruvate dehydrogenase, the rate-limiting enzyme for glucose oxidation, was increased following aging in hypertrophied hearts (Figure 6, A and C), in conjunction with an increased expression of the upstream kinases pyruvate dehydrogenase kinase 2 (PDK2) and PDK4 (Figure 6, A, D, and E). Among the known transcription factors that control PDK4 (which include E2F1, PPAR $\alpha$, forkhead box 
A

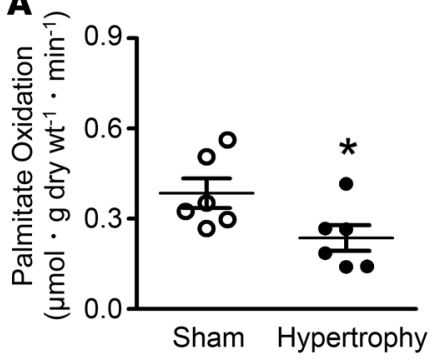

D

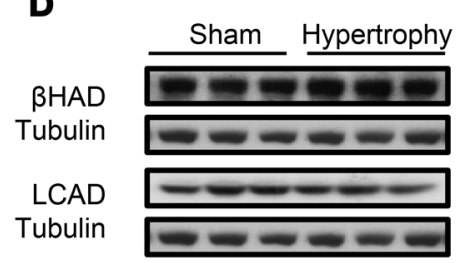

B

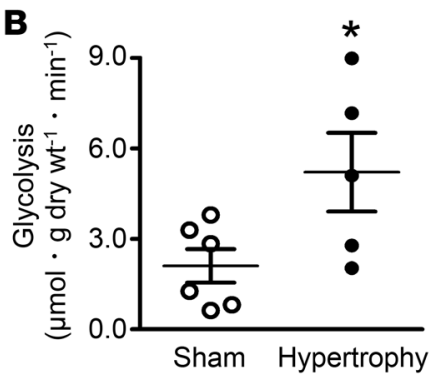

$\mathbf{E}$

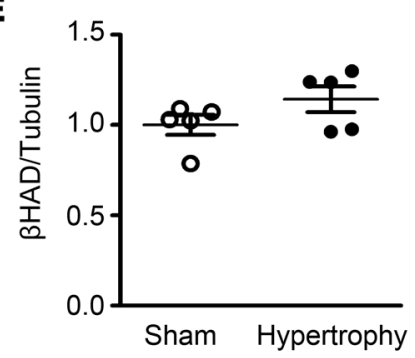

$\mathbf{E}$

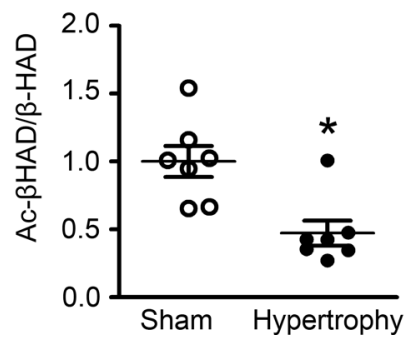

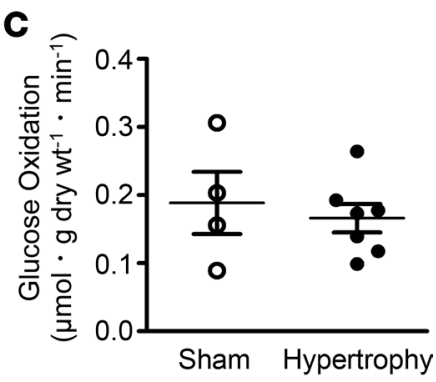

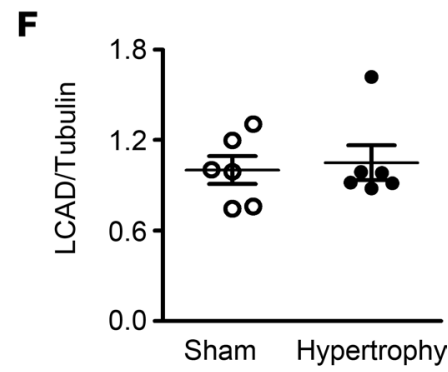

I

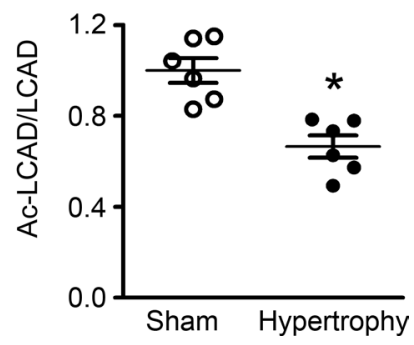

Figure 4. Acetylation and fatty acid $\beta$-oxidation in neonatal rabbit hearts with hypertrophy. (A) Rates of palmitate oxidation ( $n=6 /$ group). (B) Rates of glycolysis ( $n=5-6 /$ group). (C) Rates of glucose oxidation ( $n=4-7 /$ group). (D-F) Representative immunoblots and densitometric analysis of $\beta$-hydroxyacyl CoA dehydrogenase ( $\beta \mathrm{HAD}, n=5$ /group) and long-chain acyl CoA dehydrogenase (LCAD, $n=6 /$ group). (G-I) Representative immunoblots and densitometric analysis of acetylated $\beta \mathrm{HAD}$ (Ac- $\beta \mathrm{HAD}, n=7 /$ group) and acetylated LCAD (Ac-LCAD, $n=6$ /group). Values represent mean \pm SEM. ${ }^{*} P<0.05$ by $t$ test.

protein O1 [FOXO1], and estrogen-related receptor $\alpha$ [ERR $\alpha$, refs. 28-30), only cardiac E2F1 was enhanced postnatally in hypertrophied hearts (Figure 6, A and F). In addition, among the enzymes involved in the nuclear translocation of E2F1 (31), phosphorylation of retinoblastoma protein $(\mathrm{p}-\mathrm{Rb})$ was not altered among age groups with or without hypertrophy (Figure 6, A and G), whereas cyclin D1 and cyclin D-dependent kinase 4 (CDK4) were upregulated in hypertrophied hearts (Figure 6, A, H, and I), implicating a role of the cyclin D1/E2F1 pathway in the hypertrophy-induced upregulation of PDK4. Although glucose transporter 1 (GLUT1) and GLUT4 regulate glucose uptake and glycolysis in stressed fetal hearts (32), their protein levels were similar among age groups, regardless of the presence or absence of hypertrophy (data not shown).

GCN5L1 and PDK4 are involved in developmental changes in acetylation and energy metabolism in phenylephrineinduced cardiac hypertrophy in vitro. Since the acetyltransferase GCN5L1 was increased during maturation of human newborn hearts but was decreased in hypertrophied hearts, we examined what effect hypertrophy and Gcn5l1 gene knockdown had on fatty acid oxidation in a cardiomyocyte cell line (H9c2 cells). We conducted Gcn5l1 gene knockdown experiments using phenylephrine-stimulated (PE-stimulated) cardiac hypertrophy in undifferentiated or differentiated cardiomyocytes, a well-established in vitro model of pathological hypertrophy (33). Microscopic analysis showed that undifferentiated cells had a mononuclear and spindle shape, while differentiated cells exhibited long branched multinucleated myotubes, a distinct morphological feature of cardiomyocyte differentiation (Figure 7A). Retinoic acid-induced differentiation of H9c2 cells was also 
A

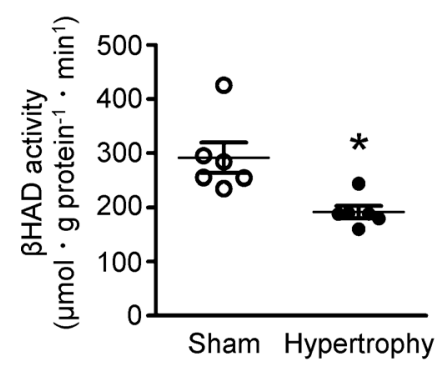

$\mathbf{E}$

GCN5L1
Tubulin
SIRT3
Tubulin

B

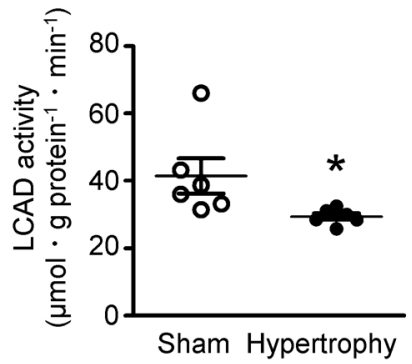

C

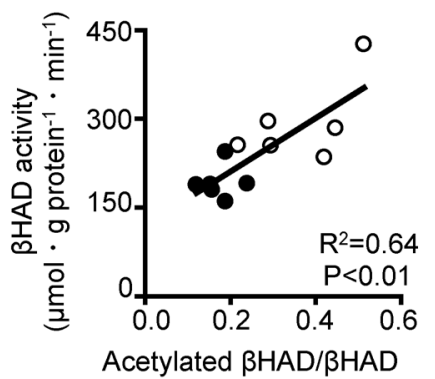

D

- Sham

- Hypertrophy

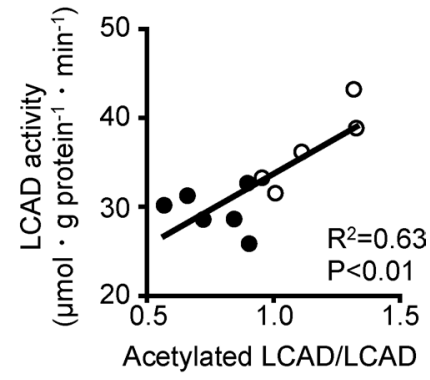

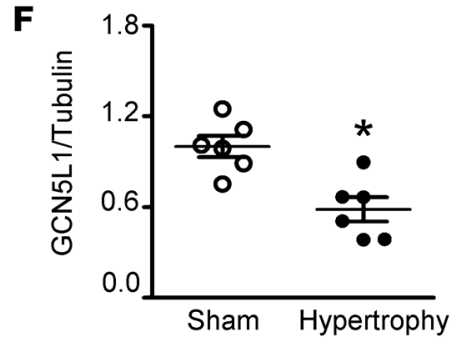

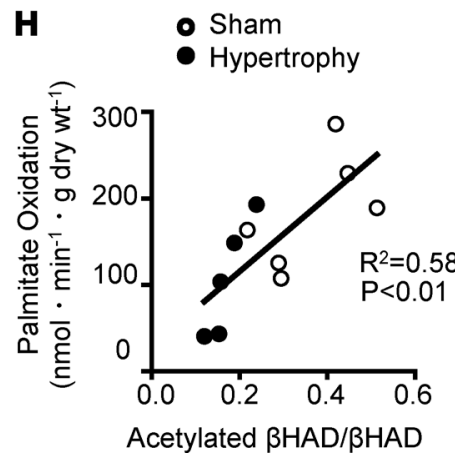

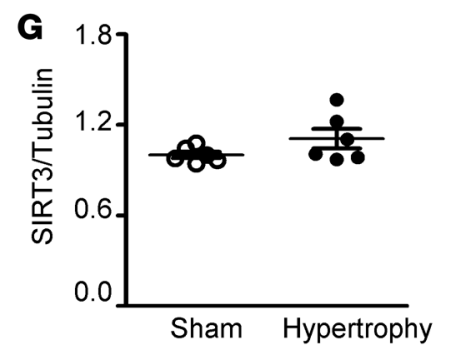

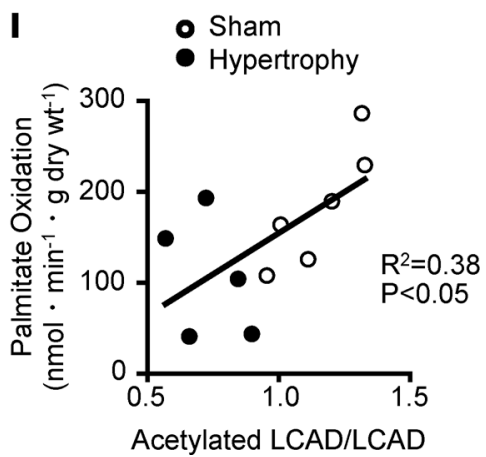

Figure 5. Positive correlation between acetylation of either $\beta$-hydroxyacyl CoA dehydrogenase or acetylation of long-chain acyl CoA dehydrogenase with either their activities or rates of palmitate oxidation, respectively, as well as expression of general control of amino acid synthesis 5-like protein 1 and sirtuin 3 in neonatal rabbit hearts with hypertrophy. (A) Activity of $\beta$-hydroxyacyl CoA dehydrogenase ( $\beta$ HAD) ( $n=6 / g r o u p)$. (B) Activity of long-chain acyl CoA dehydrogenase (LCAD) ( $n=6 /$ group). (C and D) Correlation between activity and and acetylation of $\beta$-hydroxyacyl CoA dehydrogenase (Ac- $\beta$ HAD) ( $n=6 /$ group) or acetylation of LCAD (Ac-LCAD) ( $n=5$-6/group), respectively. (E-C) Representative immunoblots and densitometric analysis of general control of amino acid synthesis 5-like protein 1 (GCN5L1) ( $n=6 /$ group) and sirtuin 3 (SIRT3) ( $n=6 /$ group). (H) Correlation between palmitate oxidation and Ac- $\beta H A D(n=6 / g r o u p)$. (I) Correlation between palmitate oxidation and Ac-LCAD ( $n=5-6 / g r o u p)$. Values represent mean $\pm \mathrm{SEM}$. ${ }^{*} P<0.05, t$ test. $\mathrm{R}^{2}$, correlation coefficient.

confirmed by increased levels of cardiac marker troponin I (Supplemental Figure 1A; supplemental material available online with this article; https://doi.org/10.1172/jci.insight.99239DS1). Additionally, H9c2 cells treated with PE displayed an increase in expression of atrial natriuretic peptide (ANP), a distinct marker of hypertrophy (Supplemental Figure 1B). The coimmunostaining with tubulin and DAPI further confirmed PE-induced hypertrophy, as indicated by increased cellular and nuclear sizes (Supplemental Figure 1, C-F).

In agreement with decreased GCN5L1 protein expression in hypertrophied human and rabbit hearts, the GCN5L1 protein level was significantly downregulated in response to PE stimulation in differentiated cells (Figure 7B). The transfection of Gcn5l1 siRNA into H9c2 cells reduced its protein expression by 20\% compared with scrambled siRNA in differentiated cells (Figure 7B). Of note, the knockdown of Gcn5ll caused cellular hypertrophy comparable to the cellular hypertrophy seen with $\mathrm{PE}$ in immature $\mathrm{H} 9 \mathrm{c} 2$ cells, whereas the hypertrophy stimulated by PE was attenuated in H9c2 cells with Gcn5l1 knockdown (Supplemental Figure 2, A and B). Furthermore, Gcn5l1 knockdown strongly induced cellular hypertrophy in the process of cell differentiation (Supplemental Figure 2, C and D). This is consistent with the phenomenon in which GCN5L1 was decreased 
A

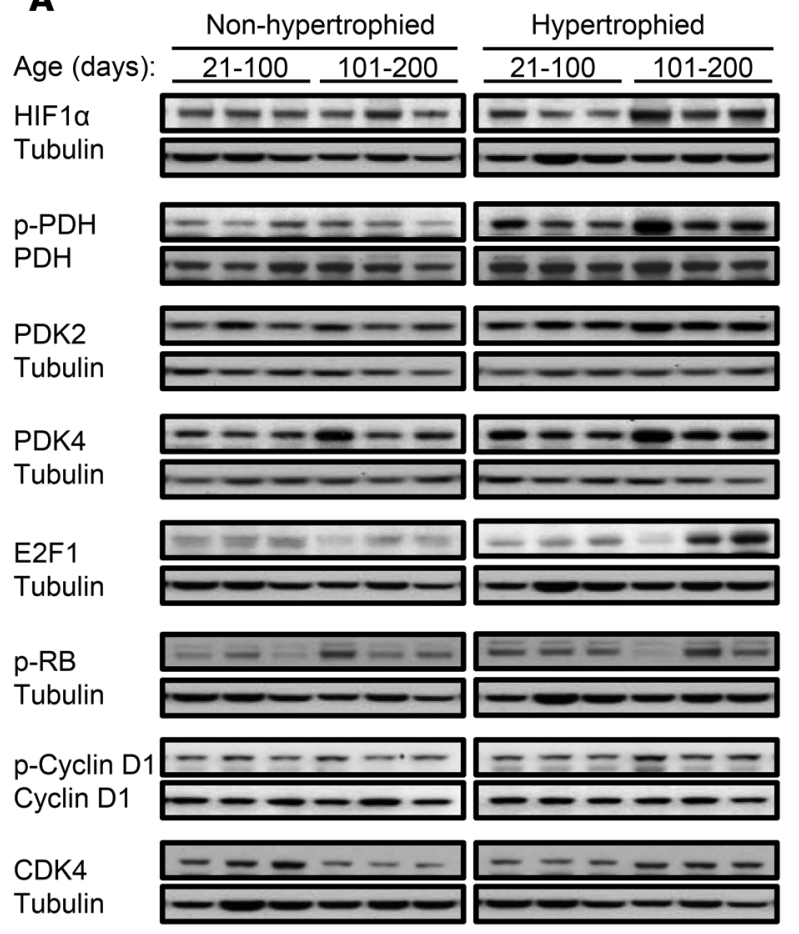

B

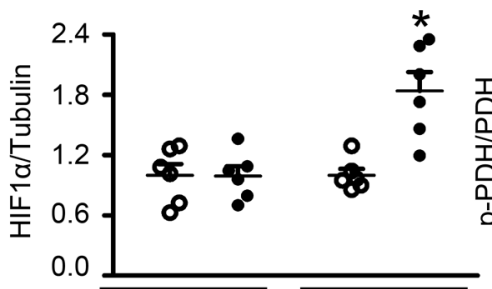

Non-hypertrophied $\overline{\text { Hypertrophied }}$
C $\quad$ Age (days): $21-100$

D

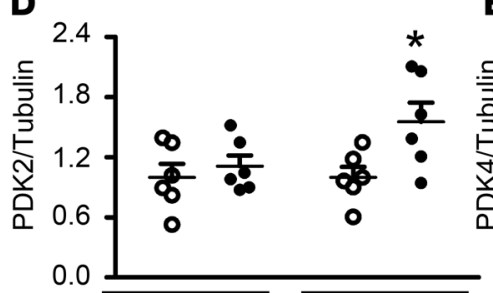

Non-hypertrophied $\overline{\text { Hypertrophied }}$

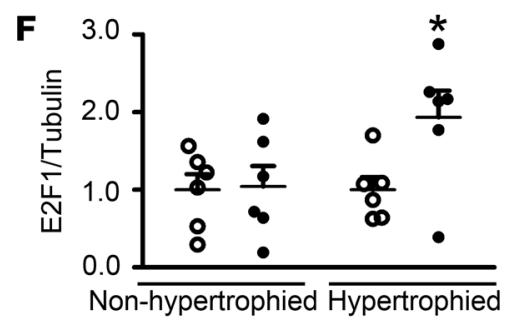

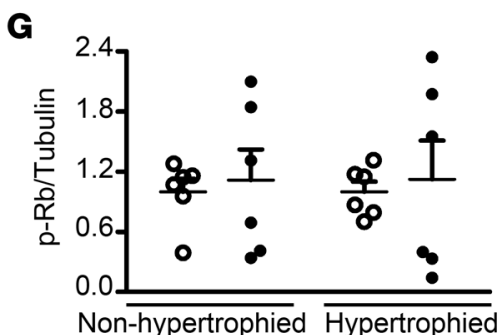
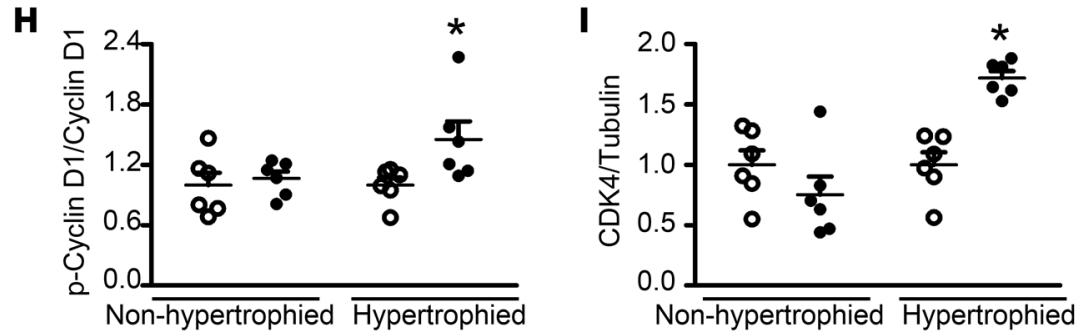

Figure 6. Upregulation of HIF1 $\alpha$ and pyruvate dehydrogenase kinase in hypertrophied human neonatal hearts. (A-E) Representative immunoblots and densitometric analysis of HIF1 $(n=6$ /group), phosphorylation of pyruvate dehydrogenase ( $\mathrm{P}-\mathrm{PDH}, n=6 /$ group), and pyruvate dehydrogenase kinase 2 (PDK2) ( $n=6$ /group) and PDK4 ( $n=6 /$ group). (F-I) Representative immunoblots and densitometric analysis of transcription factor E2F1 ( $n=6 /$ group) and phosphorylation of retinoblastoma protein ( $p-R b, n=6$ /group) as well as phosphorylation of cyclin D1 (p-cyclin D1, $n=6 /$ group) and cyclin-dependent kinase 4 (CDK4, $n=6$ /group). All lanes were run on the same gel but were noncontiguous, except for HIF1 $\alpha, p-P D H$, and E2F1, which were from different gels for each group (hypertrophy/nonhypertrophied). Values represent mean \pm SEM. ${ }^{*} P<0.05,2$-way ANOVA, comparing ages 21-100 days and 101-200 days in the same group. White circles denote age of 21-100 days, and black circles denote age of 101-200 days.

in hypertrophied human hearts (Figure 1, C and K). The repressed GCN5L1 expression due to PE stimulation or Gcn5ll knockdown was accompanied by reduced oleate oxidation rates as well as overall protein acetylation in differentiated cardiomyocytes, but this was not evident in undifferentiated cells (Figure 7, C-E).

Importantly, the acetylated levels of LCAD and $\beta \mathrm{HAD}$ were significantly suppressed following Gcn5l1 knockdown or PE stimulation in differentiated cells (Figure 8, A-C), suggesting that GCN5L1 is a key molecule modulating acetylation of myocardial fatty acid enzymes in response to hypertrophic stimuli in cardiomyocytes.

We also investigated whether PE stimulation promotes glycolysis in a PDK4-dependent manner in cardiomyocytes. Phosphorylation of PDH was upregulated in response to PE stimulation in differentiated H9c2 cells, which was significantly inhibited after incubation with a PDK4 inhibitor (Figure 8, D and E). Consistent with this result, PDK4 inhibition significantly attenuated PE-mediated increases in glycolysis in differentiated cardiomyocytes but not in undifferentiated cells (Figure 8F), suggesting that PDK4 plays a crucial role in PE-mediated upregulation of glycolysis following the development of cardiomyocytes. 
A

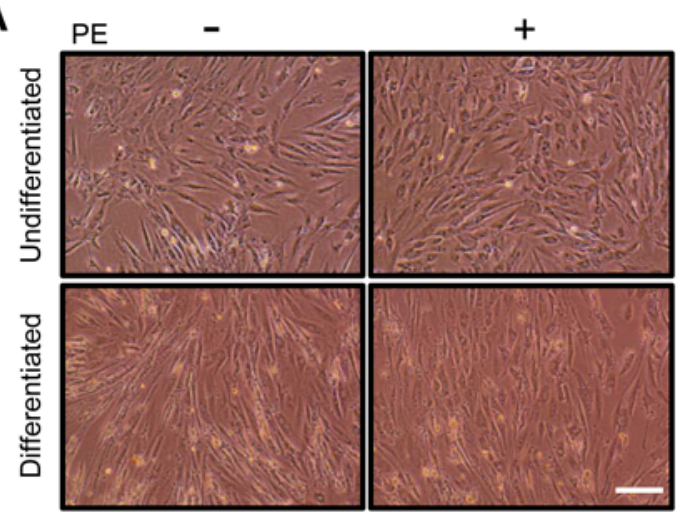

C

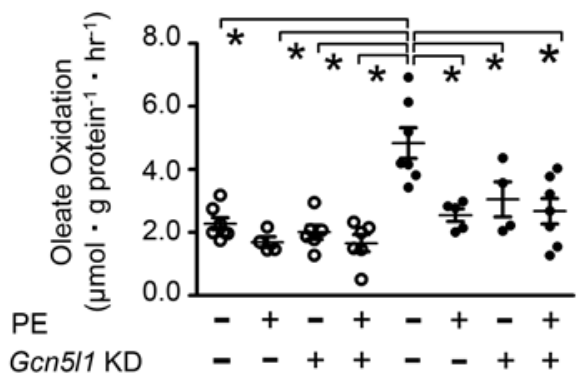

B PE
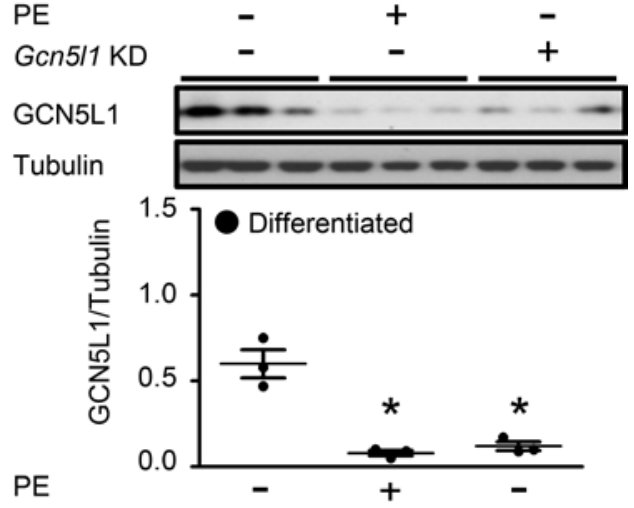

Gcn5/1 KD

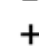

D

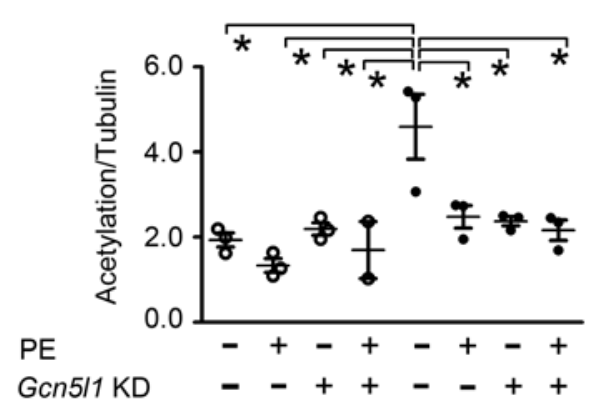

E

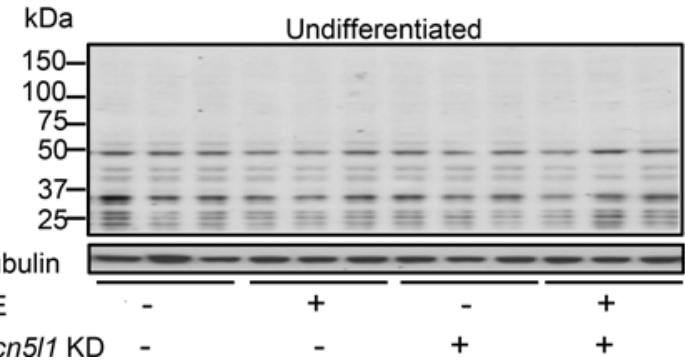

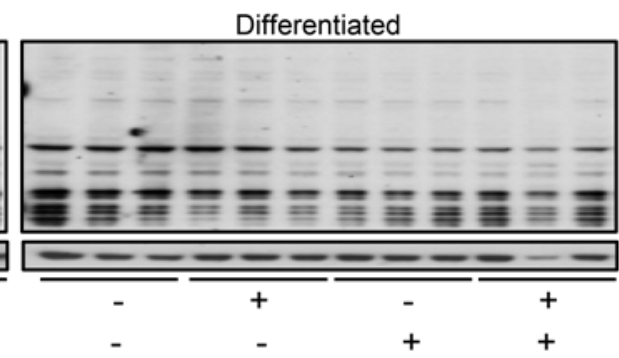

Figure 7. Expression of general control of amino acid synthesis 5-like protein 1, overall protein acetylation, and glycolysis in H9c2 cells with or without phenylephrine and knockdown of Gcn511. (A) Morphological features of H9c2 cells with or without phenylephrine (PE) (scale bar: $500 \mu \mathrm{m})$ (representative of 3 independent experiments). (B) Representative immunoblots and densitometric analysis of general control of amino acid synthesis 5-like protein 1 (GCN5L1) with/without PE and knockdown of Gcn5/1 (Gcn5/1 KD) in differentiated H9c2 cells ( $n=3 /$ group). (C) Oleate oxidation rates in H9c2 cells ( $n=4-7 /$ group). (D and E) Representative immunoblots and densitometric analysis of overall protein acetylation ( $n=3 /$ group). Values represent mean \pm SEM. ${ }^{*} P<$ 0.05, 1-way ANOVA vs. with or without Gcn5/ KD.

\section{Discussion}

Cardiac hypertrophy that occurs secondary to $\mathrm{CHD}$ is linked to the perturbation of the maturational changes in cardiac energy metabolism. Energy metabolic derangements originate from alterations in posttranslational modifications $(18)$ as well as allosteric $(7,14,15)$ and transcriptional controls of key metabolic enzymes (9). We report the finding that the presence of cardiac hypertrophy blunts protein lysine acetylation with a delayed maturation of fatty acid oxidation and an increased reliance on glycolysis as a source of energy. The mitochondrial acetyltransferase GCN5L1 plays critical role in reduced acetylation with a shift in fuel preference, as silencing of GCN5L1 resulted in a significant reduction in acetylation of fatty acid $\beta$-oxidation enzymes and cellular fatty acid oxidation rates, leading to overt hypertrophy.

Combined, these data demonstrate an important role for posttranslational lysine acetylation in controlling the dramatic energy metabolic changes in the newborn heart as well as demonstrating how the presence of hypertrophy can perturb this process.

Cardiac hypertrophy and acetylation control of fatty acid oxidation during maturation of the neonatal heart. Protein lysine acetylation has emerged as an important mechanism linking metabolic derangements to 
A

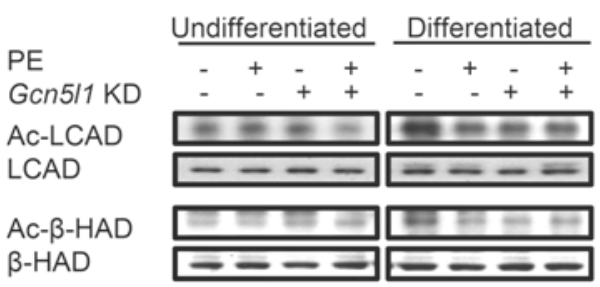

B

O Undifferentiated

Differentiated

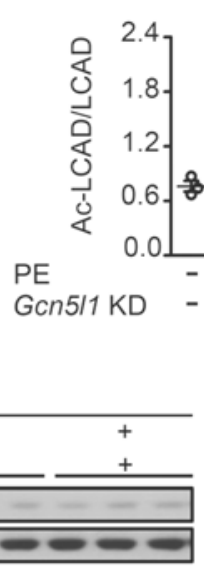

D

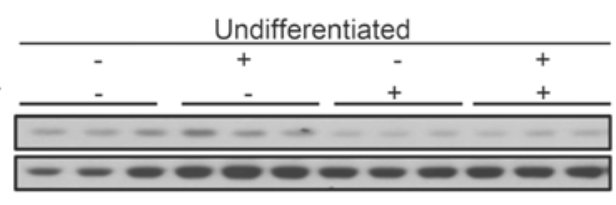

OUndifferentiated

Differentiated

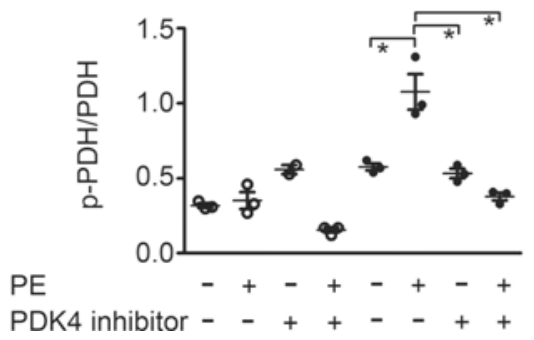

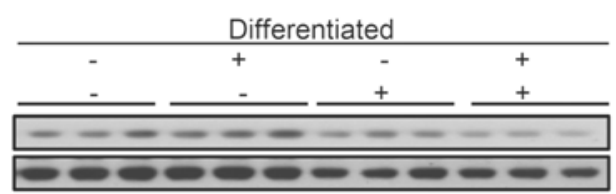

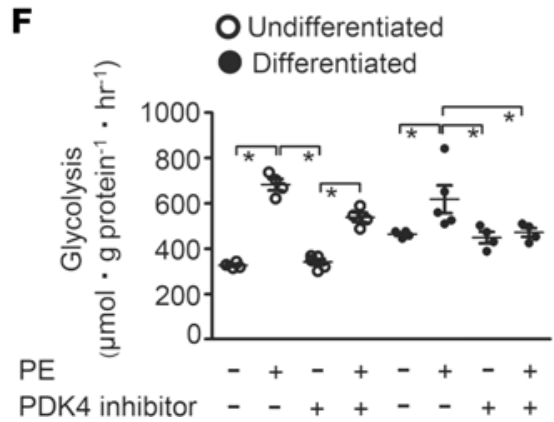

Figure 8. Changes in acetylation and glycolysis in H9c2 cells with or without phenylephrine and knockdown of general control of amino acid synthesis 5-like protein 1 or inhibition of pyruvate dehydrogenase kinase 4. (A-C) Representative immunoblots and densitometric analysis of acetylated long-chain acyl CoA dehydrogenase (Ac-LCAD, $n=3 /$ group) and acetylated $\beta$-hydroxyacyl CoA dehydrogenase (Ac- $\beta H A D, n=3 /$ group). (D and E) Representative immunoblots and densitometric analysis of phosphorylated pyruvate dehydrogenase ( $\mathrm{p}-\mathrm{PDH}, n=3$ /group) with or without pyruvate dehydrogenase kinase 4 (PDK4) inhibitor in H9c2 cells. (F) Glycolysis rates with/without phenylephrine (PE) and PDK4 inhibitor in H9c2 cells ( $n=4-5 / g r o u p)$. All lanes were run on the same ge but were noncontiguous, except for Ac-LCAD and Ac- $\beta H A D$, which were from different gels for each group (hypertrophy/nonhypertrophied). Values represent mean $\pm S E M$. ${ }^{*} P<0.05$, 1-way ANOVA vs. without or without knockdown of general control of amino acid synthesis 5-like protein 1 (Gcn5/1 KD).

pathological remodeling of the heart in obesity, diabetes, and heart failure $(16,34,35)$. We and others have recently demonstrated that increased acetylation of fatty acid $\beta$-oxidation enzymes promotes their activities and thereby increases fatty acid utilization in obese or diabetic hearts, which is responsible for metabolic inflexibility $(16,17,22)$. In addition to the pathological role, we recently showed that cardiac acetylation contributes to the postnatal metabolic shift from glycolysis to fatty acid oxidation in the newborn rabbit hearts (16). In agreement with this, the present study also shows an age-dependent increase in overall acetylation as well as acetylated LCAD and $\beta \mathrm{HAD}$ in nonhypertrophied hearts. Importantly, the presence of cardiac hypertrophy abrogates a physiological increase in acetylation in the neonatal heart, resulting in delayed maturation of fatty acid oxidation, which is in line with our previous finding (8). Of interest, the decreased mitochondrial fatty acid oxidation is linked to the increased enzymes involved in triacylglyceride and ceramide biosynthesis in hypertrophied hearts. This may reflect the lipid accumulation within hypertrophied cardiomyocytes, implicating its role in the development of insulin resistance and cardiac dysfunction $(36,37)$. However, further investigation for actual lipid contents is necessary to confirm this possibility.

We previously reported that the combination of delayed fatty acid oxidation with low glucose oxidation compromises the postischemic recovery of neonatal hypertrophied hearts, and stimulating fatty acid $\beta$-oxidation with a PPAR $\alpha$ agonist had beneficial effects on post-ischemic functional recovery of these hearts (9). As such, hypertrophy-induced alterations in cardiac energy metabolism may increase the susceptibility to ischemic injury during corrective surgery for CHDs. 
Our data appear to be in contrast to studies that suggest increased acetylation provokes mitochondrial dysfunction and energy deficiency in failing hearts $(34,35)$. However, the postnatal change in cardiac acetylation has not been explored previously. In addition, the effect of acetylation on metabolic enzymes might be different between RV and LV tissues in response to volume and/or pressure overload because the degree of acetylation is shown to correlate with the inherent contractility of failing myocardium (38). Hence, it seems difficult to translate our findings to other types of cardiac hypertrophy.

The level of protein acetylation is regulated by the balance between acetyltransferase and deacetylase enzymes $(19,20)$. In the adult heart, the deacetylase, SIRT3, has an important role in regulating cardiac fatty acid oxidation (19). Fatty acid oxidative enzymes are a major target for SIRT3 (19). Furthermore, decreasing SIRT3 expression (by either knocking out SIRT3 or feeding mice a high-fat diet) increases acetylation of $\mathrm{LCAD}$ and $\beta \mathrm{HAD}$, resulting in an increase in fatty acid oxidation (17). However, in human newborn hearts, the increased acetylation with age was not accompanied by alterations in SIRT3 expression. Curiously, an increase in SIRT3 expression was observed with increased age in hypertrophied newborn hearts and, combined with the decrease in GCN5L1 expression, may contribute to the decreased overall acetylation occurring at the same time with decreased LCAD and $\beta \mathrm{HAD}$ acetylation that was seen in the hypertrophied hearts. However, the similar relationship between SIRT3 and fatty acid $\beta$-oxidation enzymes was not observed in rabbit hypertrophied hearts, highlighting the need for further study the role of SIRT3 in this pathway.

However, the important role of GCN5L1 in mediating these acetylation changes was supported in the isolated cardiomyocyte studies, since inhibition of GCN5L1 significantly attenuated the levels of acetylated $\mathrm{LCAD}$ and $\beta \mathrm{HAD}$, resulting in a depression of fatty acid oxidation rates. Notably, in vitro studies with the manipulation of GCN5L1 clearly demonstrate that the repressed GCN5L1 is a critical contributor for cardiac hypertrophy, corroborating the reduced GCN5L1 expression observed in human hypertrophied hearts. We previously reported that the mitochondrial acetyltransferase GCN5L1 is postneonatally elevated in the newborn heart and facilitates fatty acid oxidation by enhancing mitochondrial protein acetylation (16). This is further supported in the current study, suggesting that hypertrophy-induced downregulation of GCN5L1 contributes to a decrease in acetylation of fatty acid $\beta$-oxidation enzymes, which, in turn, inhibits a maturational increase in fatty acid oxidation in neonatal hearts and further exacerbates cardiac hypertrophy.

Acetylation control of mitochondrial biogenesis. Aside from its role as a acetyltransferase, recent studies have shown that GCN5L1 positively regulates mitochondrial content in concert with PGC1 $\alpha$, a master regulator of mitochondrial biogenesis $(39,40)$. In the present study, a postnatal increase in GCN5L1 was associated with an increase in CS activity, a well-defined marker of mitochondrial content in nonhypertrophied CHD hearts, while CS activity was unaltered in hypertrophied hearts with a marked decline in GCN5L1. Moreover, this decrease in mitochondrial content is potentially due to an increase in acetylation of PGC1 $\alpha$, because hyperacetylation of PGC1 $\alpha$ can inhibit enzyme activity in skeletal muscles and newborn hearts $(16,41)$. Although both SIRT1 and SIRT6 have been shown to coordinately modulate acetylation of PGC1 $\alpha(42)$, both deacetylases are unlikely to contribute to the increase in acetylation of PGC1 $\alpha$ in hypertrophied hearts. Instead, protein expression of GCN5, a nuclear acetyltransferase and a downstream target of SIRT6, is reduced in nonhypertrophied hearts, indicating the relative increase in PGC1 $\alpha$ acetylation through sustained GCN5 expression in hypertrophied hearts. Collectively, hyperacetylation of PGC1 $\alpha$ together with the depletion of GCN5L1 may contribute to impaired mitochondrial biogenesis in hypertrophied hearts with CHD.

PDK4 axis is upregulated and contributes to increase in glycolysis in hypertrophied hearts. PDK4 negatively regulates glucose oxidation through phosphorylation and inhibition of $\mathrm{PDH}$ (43). In adult hearts, we previously reported that hypertrophy-induced activation of PDK4 plays a critical role in exerting a cardiac metabolic switch from glucose to fatty acid oxidation (44). In line with this, Piao et al. showed that PDK4 activation contributes to an increase in glycolysis relative to glucose oxidation in a rodent model of RV hypertrophy (29). In this study, we show that in hypertrophied neonatal hearts the increase in PDK4 expression and phosphorylation were accompanied by an increased reliance on glycolysis as a source of ATP production. Although the PDK4 promoter region has multiple binding sites for ERR $\alpha /$ PPAR $\alpha$ and FOXO1 (28-30), those transcriptional regulators are unlikely to initiate PDK4 activation. Instead, the cyclin D1/E2F pathway is operational in stimulating PDK4 in hypertrophied CHD hearts. This is in agreement with our previous findings that angiotensin II induces a reduction in glucose oxidation via the cyclin D1/CDK4/E2F1 axis (33). As with CHD hearts, this pathway has also been reported to be increased in, and correlated to, cardiomyocyte hypertrophy in myocardial samples from patients with advanced heart failure (45). In addition to E2F1-induced PDK4 activation, a postnatal increase in 
HIF1 $\alpha$, a key transcriptional regulator of genes that favor anaerobic glycolysis, potentially contributes to the glycolytic metabolic profile in hypertrophied neonatal hearts $(8,46)$. As the heart matures and the availability of oxygen rises, HIF-1 $\alpha$ protein levels rapidly drop, resulting in a decreased reliance on glycolysis (46). However, in the presence of hypertrophy, we and other groups observed an increase in HIF1 $\alpha$ expression during the development and progression of hypertrophy $(8,47)$. The glycolytic phenotype seen in hypertrophied hearts appears to be a maladaptive response, because dichloroacetate, a nonspecific PDK inhibitor, decreases lactate levels and improves cardiac function in an experimental model of hypertrophy (48). Indeed, a potent PDK4 inhibitor reduced PE-induced PDH phosphorylation, which results in reduced glycolytic rates in hypertrophied cardiomyocytes. As a result, inhibition of PDK4 might be a potential therapy for cardiac hypertrophy associated with CHD.

The limitation of the current study is that clinical population is not representative of the normal neonate. The lack of a normal control population can be responsible for the lack of significant differences for some of the proteins among the groups and, most importantly, can affect the generalizability of such findings to the whole neonatal population. Second, assessment of the cardiac energetic profile and immunoblotting were performed only on the RV. Although animal studies suggest the presence of ventricle-specific differences in energy metabolism, due to ethical issues, this study was only able to analyze RV biopsy samples that were resected as part of a standard surgical procedure. The newborn heart samples examined in this study originated from infants from 21-100 days of age and 101-200 days of age. We also collected myocardial samples from newborns of 0-21 days of age, where key metabolic changes may be occurring. However, most of these infants in this age group were infants with hypoplastic left heart syndrome. Since we were unable to accurately access the presence or absence of hypertrophy in these infants (indeed, more were hypertrophied), we did not include any infants with hypoplastic left heart syndrome in this study. Finally, limited tissue availability prevented additional biochemical analyses beyond determination of the expression of some of the major proteins as well as analysis of mRNA expression involved in the energy substrate metabolism. However, data obtained from such analysis are strong enough to support some of the experimental findings from animal models and to further enhance the research efforts in this area.

In conclusion, an increased acetylation of fatty acid $\beta$-oxidation enzymes contributes to the dramatic increase in cardiac fatty acid oxidation rates after birth. The presence of cardiac hypertrophy prevents the normal increase in myocardial acetylation after birth, resulting in a delayed maturation of fatty acid oxidation. The low rates of fatty acid oxidation combined with low rates of glucose oxidation in the hypertrophied newborn heart compromise the energy-generating capacity of the heart and increase the susceptibility of the heart to ischemic injury, as is seen during cardiac surgery to correct CHDs.

\section{Methods}

Newborn subjects. A total of 84 RV samples were collected from infants (21-200 days) undergoing corrective surgery for CHDs at the University of Alberta Hospital (Edmonton, Alberta, Canada) between 2006 and 2016. Myocardial samples (5-200 mg) were obtained as part of the normally resected and discarded specimen from the RV and stored immediately in liquid nitrogen until used for biochemical analyses. Infants with CHDs had one or more cardiac defects, including tetralogy of Fallot, ventricular septal defect, atrioventricular septal defect, double outlet right ventricle, transposition of the great arteries, tricuspid atresia, pulmonary atresia, and bicuspid aortic valve. Body surface area was calculated according to the Haycock formula (49). Since dramatic maturational changes in cardiac energy metabolism are reported to occur after birth $(12,16,50)$, myocardium samples were stratified in two age groups based on the age of the patient: age group 1 (21-100 days) and age group 2 (101-200 days). Myocardial samples were further stratified based on the presence or absence of RV hypertrophy. In order to better define the RV hypertrophy, a Z-score of RVAW was determined according to a previous report (51). In brief, RVAW at the end diastole was measured via M-mode and 2D echocardiographic techniques by using multiple apical and parasternal long axis views from presurgical recordings (median 2-3 recordings in the immediate days preceding surgery), and a $Z$-score of RVAW was calculated based on body surface area (51). RV hypertrophy was defined as a $Z$-score of RVAW greater than 2.0, which corresponds to 2 SDs above the mean value for a particular body surface area $(52,53)$. LV enddiastolic diameter, LV fractional shortening, LV mass index, RV end-diastolic diameter, and tricuspid annular plane systolic excursion were also assessed by echocardiography. 
Animal studies. Newborn New Zealand White rabbits of either sex (7 days old, 90-200 g) (Charles River Co.) were subjected to an aortocaval shunt to induce volume-overloaded cardiac hypertrophy under anesthesia with inhaled isofluorane $(2 \%)$, as described previously $(8,9)$. Fourteen days after the surgery, all animals were euthanized with sodium pentobarbital $(60 \mathrm{mg} / \mathrm{kg}$ body weight), and hearts were removed for isolated biventricular working heart perfusions.

Biventricular working heart perfusion. Rabbit hearts were perfused using a modified Krebs-Henseleit solution that contains $2.5 \mathrm{mmol} / 1 \mathrm{Ca}^{2+}, 5.5 \mathrm{mmol} / 1$ glucose, $1.2 \mathrm{mmol} / 1$ palmitate prebound to $3 \% \mathrm{BSA}$, $0.5 \mathrm{mmol} / 1$ lactate, and $100 \mu \mathrm{U} / \mathrm{ml}$ insulin, as described previously $(8,9)$. Rates of palmitate oxidation, glycolysis, and glucose oxidation were measured during the aerobic periods using radiolabeled $\left[9,10-{ }^{3} \mathrm{H}\right]$ palmitate, $\left[5-{ }^{3} \mathrm{H}\right]$ glucose, and $\left[\mathrm{U}-{ }^{14} \mathrm{C}\right]$ glucose, respectively. Heart rates and peak systolic pressures of aortas and pulmonary veins were measured using a Gould P21 pressure transducer attached to the aortic and pulmonary outflow line. Normalized cardiac function was calculated as [heart rates $\times$ (aortic peak systolic pressure + pulmonary vein peak systolic pressure)], as previously described $(8,9)$. At the end of the aerobic perfusion protocol, hearts were immediately frozen in liquid $\mathrm{N}_{2}$ and stored at $-80^{\circ} \mathrm{C}$. Steady-state rates of ATP production from exogenous substrates was calculated with the values of 2, 31, and $105 \mathrm{~mol} \mathrm{ATP} / \mathrm{mol}$ of glucose produced from glycolysis, glucose oxidation, and palmitate oxidation, respectively (8).

Immunoblot analysis. Thirty $\mathrm{mg}$ frozen RV tissue was homogenized for 30 seconds with a Polytron homogenizer in buffer containing $50 \mathrm{mmol} / 1$ Tris- $\mathrm{HCl}, 150 \mathrm{mmol} / 1 \mathrm{NaCl}, 0.5 \% \mathrm{NP}-40,1 \%$ Triton-X 100, $5 \mathrm{mmol} / 1 \mathrm{EDTA}$, and $0.1 \%$ SDS in the presence of phosphatases and protease inhibitors (MilliporeSigma) as well as deacetylase inhibitors ( $10 \mathrm{mmol} / 1$ nicotinamide, $10 \mu \mathrm{mol} / 1$ trichostatin $\mathrm{A}$, and 10 mmol/1 sodium butyrate). Thirty $\mu \mathrm{g}$ of the denatured proteins was subjected to $5 \%-12 \%$ SDS/PAGE and transferred to nitrocellulose membranes as previously described (54). After blocking in 5\% fat-free milk for 1 hour, membranes were probed with the following antibodies: acetyl-lysin (catalog 9441), PDH (catalog 2784), phosphor-Rb (catalog 9307), phospho-cyclin D1 (catalog 3300), cyclin D1(catalog 2922), CDK4 (catalog 12790), phosphor-FOXO1 (catalog 9461), FOXO1 (catalog 9454), GLUT4 (catalog 2213), and ATGL (catalog 2439) (all from Cell Signaling Technology); SIRT1 (catalog ab7343), SIRT2 (catalog ab75436), SIRT3 (catalog ab86671), SIRT4 (catalog ab124521), SIRT5 (catalog ab13697), PDK4 (catalog ab71240), PPAR $\alpha$ (catalog ab8934), PGC1 $\alpha$ (catalog ab77210), ERR $\alpha$ (catalog ab93173), LCAD (catalog ab129711), BHAD (catalog ab37673), E2F1 (catalog ab86431), and ANP (catalog ab189921) (all from Abcam); phosphor-PDH serine 293 (catalog ABS204) and SIRT6 (catalog S4322) (both from MilliporeSigma); HIF1 $\alpha$ (catalog NB100-449) and DGAT2 (catalog NB100-57851) (both from Novus Biologicals); GCN5 (catalog sc-20698), SPT1 (catalog sc-32916), SPT2 (catalog sc-27500), GLUT1(catalog sc-1605), and PDK2 (catalog sc-100534) (all from Santa Cruz); and GCN5L1 (provided by M.N. Sack, NIH, Bethesda, Maryland, USA). Membranes were incubated with the appropriate secondary antibodies (goat anti-rabbit, catalog sc-2054; goat anti-mouse, catalog sc-2055; and goat anti-chicken, catalog sc-2901; Santa Cruz) for 1 hour. These bands were visualized with enhanced chemiluminescence and quantified with ImageJ software (NIH). Tubulin (catalog 2144; Cell Signaling Technology) was used as an internal control to normalize for any variation in protein loading between samples.

Immunoprecipitation. A total of $300 \mu \mathrm{g}$ of lysates was precleared with $20 \mu \mathrm{l}$ protein A/G-agarose beads. The lysates were incubated with anti-acetyl-lysine antibodies $(3 \mu \mathrm{g} / 300 \mu \mathrm{g}$ lysate, catalog 9441; Cell Signaling Technology) overnight at $4^{\circ} \mathrm{C}$, and $40 \mu \mathrm{l}$ protein $\mathrm{A} / \mathrm{G}$-agarose beads was added to each sample and incubated on a rotator for 6 hours at $4^{\circ} \mathrm{C}$ as previously described $(16,17)$. After 6 hours, samples were washed 3 times and centrifuged at $16,000 \mathrm{~g}$ for 5 minutes. The immune complexes were then subjected to immunoblot analysis as described above. As a negative control, heart lysates were immunoprecipitated with normal rabbit IgG (sc-2027; Santa Cruz) coupled to agarose A/G beads or agarose A/G beads alone. Heart lysates without rabbit IgG were used as a positive control. The light chain of $\mathrm{IgG}$ or heavy chain of IgG was used as the loading control.

Measurement of $\beta H A D$ activity, LCAD activity, and CS activity. $\beta \mathrm{HAD}$ activity was measured in total heart lysates prepared from frozen heart tissues, as previously described (54). Briefly, heart lysates were pipetted into a 96-well plate that contains $50 \mathrm{mmol} / 1$ imidazole and $150 \mathrm{mmol} / 1 \mathrm{NADH}$ as an assay buffer. The reaction was initiated by the addition of acetoacetyl CoA $(100 \mathrm{mmol} / \mathrm{l})$, and the absorbance was followed at a $340-\mathrm{nm}$ wavelength for 5 minutes. LCAD activity was measured by following the initial decrease in absorbance at $300 \mathrm{~nm}$ upon the reduction of the ferricenium ion measured as previously described (17). The reaction was started by the addition of palmitoyl CoA $(50 \mu \mathrm{mol} / 1)$, followed spectrophotometrically for 2 minutes. CS activity was determined in the mitochondria isolated from heart tissues and was mea- 
sured with buffer containing $0.2 \mathrm{mmol} / 1$ acetyl CoA, $0.5 \mathrm{mmol} / 1$ oxaloacetate in $100 \mathrm{mmol} / 1 \mathrm{Tris}, \mathrm{pH} 7.5$, buffer, and $0.1 \mathrm{mmol} / 1$ and 5'-Dithiobis 2-nitrobenzoic acid, followed spectrometrically at $412 \mathrm{~nm}$ (55). Those enzyme activities were normalized for mg protein content.

Cell culture, transfection, and immunostaining. Embryonic rat heart-derived H9c2 cells (ATCC) were cultured in Dulbecco's Modified Eagles Medium (MilliporeSigma) with 10\% (v/v) FBS and 1\% (w/v) PenStrep (MilliporeSigma) at $37^{\circ} \mathrm{C}$ with a humidified atmosphere of $5 \% \mathrm{CO}_{2}$. The $\mathrm{H} 9 \mathrm{c} 2$ cardiomyoblasts were seeded into the $25-\mathrm{cm}^{2}$ flasks at a density of $5 \times 10^{3}$ cells $/ \mathrm{ml}$; when they reached approximately $70 \%-80 \%$ confluency, the myoblasts were divided into two groups: undifferentiated cardiac myoblasts and differentiated cardiomyocytes. Cell cardiac differentiation was induced with medium containing $1 \%$ FBS followed by the supplementation of $1 \mu \mathrm{mol} / 1$ all-trans-retinoic acid (MilliporeSigma) for 7-10 days, as previously described $(56,57)$. The differentiation was confirmed by morphological features by light microscopy, including alignment, elongation, fusion, and protein expression of cardiac marker troponin I, as previously described $(56,57)$. To induce hypertrophy, undifferentiated or differentiated cardiomyocytes were randomly grouped and exposed to either saline or $100 \mathrm{nmol} / 1 \mathrm{PE}$ for 48 hours. These cells were also seeded in a 6 -well plate at a density of $2.0 \times 10^{5}$ cells/well and transfected 24 hours later with $50 \mathrm{nmol} / 1$ control scrambled siRNA or siRNA against Gcn5l1 for 48 hours using DharmaFECT reagent (Dharmacon) according to the manufacturer's instructions (16). For the inhibition of PDK4, H9c2 cells were incubated at $37^{\circ} \mathrm{C}$ with $100 \mu \mathrm{mol} / 1$ PDK4 inhibitor (CR-37-001, molecular weight 158.2; MilliporeSigma) for 24 hours. For immunofluorescence staining, cells plated onto coverslips were fixed with $4 \%$ paraformaldehyde in PBS solution, followed by the permeabilization with $0.1 \%$ Triton X-100. After washing with PBS and blocking with 3\% BSA for 1 hour, cells were incubated with primary antibody ( $\alpha$-Tubulin; Cell Signaling Technology) overnight at $4^{\circ} \mathrm{C}$, followed by incubation with Dako Envision HRP labelled polymer anti-rabbit for 30 minutes. The coverslips were mounted on glass slides in Vectashield mounting medium containing DAPI (Vector Laboratories) and visualized by fluorescence microscopy.

Measurements of fatty acid oxidation and glycolytic rates of cardiomyocytes. Oleate oxidation rates were measured in $\mathrm{H} 9 \mathrm{c} 2$ cells grown in T25 flasks as previously described $(16,58)$. Briefly, cell culture media were switched to Krebs-Henseleit buffer containing $118 \mathrm{mmol} / 1 \mathrm{NaCl}, 4.7 \mathrm{mmol} / 1 \mathrm{KCl}, 1.2 \mathrm{mmol} / 1 \mathrm{KH}_{2} \mathrm{PO}_{4}, 1.2 \mathrm{mmol} / 1$ $\mathrm{MgSO}_{4}, 2.5 \mathrm{mmol} / 1 \mathrm{CaCl}_{2}, 25 \mathrm{mmol} / 1 \mathrm{NaHCO}_{3} 5 \mathrm{mmol} / 1$ glucose, $4 \% \mathrm{BSA}$, and $0.4 \mathrm{mmol} / 1\left[1-{ }^{14} \mathrm{C}\right]$ oleate. After the incubation for 3 hours at $37^{\circ} \mathrm{C}$, oleate oxidation rates were measured by counting ${ }^{14} \mathrm{CO}_{2}$ captured into hyamine hydroxide in a scintillation counter (2800TR; Perkin Elmer). Glycolytic rates were assessed using cells incubated with $\left[5-{ }^{3} \mathrm{H}\right]$ glucose, and the $3 \mathrm{H}_{2} \mathrm{O}$ released at the enolase step of glycolysis was measured (58).

Statistics. Data are represented as mean \pm SEM. The significance of differences among age groups in hypertrophied or nonhypertrophied hearts was estimated by 2-way ANOVA followed by the Bonferroni post hoc tests. For multiple group comparisons, 1-way ANOVA followed by Tukey's test was performed. For categorical variables, the $\chi^{2}$ or Fisher exact tests were used for further analysis. Correlations were examined by linear regression analysis using the least squares method. A 2-tailed $t$ test was used. The differences were considered statistically significant when $P$ values were less than 0.05 .

Study approval. Collection of specimens from newborn patients were approved by the University of Alberta Health Research Ethics Board protocol (ID no. Pro0001112). All participants or their guardians provided written informed consent for the sample collection, following analysis and prior to inclusion in the study. All animals were treated according to the guidelines of the Canadian Council on Animal Care. All animal protocols were approved by the University of Alberta Health Sciences Animal Welfare Committee.

\section{Author contributions}

$\mathrm{AF}$ and LZ contributed to the design of the study, performed experiments, and drafted the manuscript. $\mathrm{AH}$, SR, and TA contributed to data searching and characterization of human heart samples. VHL and CSW contributed to data for the rabbit heart perfusions. KKD, LKH, PFK, and IMR contributed to collection of human newborn biopsies and were involved in the manuscript editing. GDL contributed to the original project design and the drafting of the manuscript.

\section{Acknowledgments}

This work was supported by a grant from the Canadian Institutes of Health Research to GDL and IMR and Woman and Children's Health Research Institute grants to PFK and GDL. GDL was an Alberta Innovates - Health Solutions Scientist. AF was supported by a fellowship grant from the Manpei Suzuki 
Diabetes Foundation and the Japan Ministry of Education, Science, and Culture (17K10137).

Address correspondence to: Gary D. Lopaschuk, 423 Heritage Medical Research Building, University of Alberta, Edmonton, Alberta T6G 2S2, Canada. Phone: 780.492.2170; Email: gary.lopaschuk@ualberta.ca.

1. Marelli AJ, Mackie AS, Ionescu-Ittu R, Rahme E, Pilote L. Congenital heart disease in the general population: changing prevalence and age distribution. Circulation. 2007;115(2):163-172.

2. Khairy P, Ionescu-Ittu R, Mackie AS, Abrahamowicz M, Pilote L, Marelli AJ. Changing mortality in congenital heart disease. J Am Coll Cardiol. 2010;56(14):1149-1157.

3. Norozi K, et al. Incidence and risk distribution of heart failure in adolescents and adults with congenital heart disease after cardiac surgery. Am J Cardiol. 2006;97(8):1238-1243.

4. Verheugt CL, et al. Mortality in adult congenital heart disease. Eur Heart J. 2010;31(10):1220-1229.

5. Iacobazzi D, Suleiman MS, Ghorbel M, George SJ, Caputo M, Tulloh RM. Cellular and molecular basis of RV hypertrophy in congenital heart disease. Heart. 2016;102(1):12-17.

6. Reddy S, Bernstein D. Molecular mechanisms of right ventricular failure. Circulation. 2015;132(18):1734-1742.

7. Kantor PF, Robertson MA, Coe JY, Lopaschuk GD. Volume overload hypertrophy of the newborn heart slows the maturation of enzymes involved in the regulation of fatty acid metabolism. J Am Coll Cardiol. 1999;33(6):1724-1734.

8. Oka T, et al. Cardiac hypertrophy in the newborn delays the maturation of fatty acid $\beta$-oxidation and compromises postischemic functional recovery. Am J Physiol Heart Circ Physiol. 2012;302(9):H1784-H1794.

9. Lam VH, et al. Activating PPAR $\alpha$ prevents post-ischemic contractile dysfunction in hypertrophied neonatal hearts. Circ Res. 2015;117(1):41-51.

10. Lopaschuk GD, Spafford MA, Marsh DR. Glycolysis is predominant source of myocardial ATP production immediately after birth. Am J Physiol. 1991;261(6 Pt 2):H1698-H1705.

11. Girard J, Ferré P, Pégorier JP, Duée PH. Adaptations of glucose and fatty acid metabolism during perinatal period and sucklingweaning transition. Physiol Rev. 1992;72(2):507-562.

12. Lopaschuk GD, Collins-Nakai RL, Itoi T. Developmental changes in energy substrate use by the heart. Cardiovasc Res. 1992;26(12):1172-1180.

13. Lehman JJ, Barger PM, Kovacs A, Saffitz JE, Medeiros DM, Kelly DP. Peroxisome proliferator-activated receptor gamma coactivator-1 promotes cardiac mitochondrial biogenesis. J Clin Invest. 2000;106(7):847-856.

14. Dyck JR, Barr AJ, Barr RL, Kolattukudy PE, Lopaschuk GD. Characterization of cardiac malonyl-CoA decarboxylase and its putative role in regulating fatty acid oxidation. Am J Physiol. 1998;275(6 Pt 2):H2122-H2129.

15. Lopaschuk GD, Witters LA, Itoi T, Barr R, Barr A. Acetyl-CoA carboxylase involvement in the rapid maturation of fatty acid oxidation in the newborn rabbit heart. J Biol Chem. 1994;269(41):25871-25878.

16. Fukushima A, et al. Acetylation and succinylation contribute to maturational alterations in energy metabolism in the newborn heart. Am J Physiol Heart Circ Physiol. 2016;311(2):H347-H363.

17. Alrob OA, et al. Obesity-induced lysine acetylation increases cardiac fatty acid oxidation and impairs insulin signalling. Cardiovasc Res. 2014;103(4):485-497.

18. Fukushima A, Lopaschuk GD. Acetylation control of cardiac fatty acid $\beta$-oxidation and energy metabolism in obesity, diabetes, and heart failure. Biochim Biophys Acta. 2016;1862(12):2211-2220.

19. Hirschey MD, et al. SIRT3 regulates mitochondrial fatty-acid oxidation by reversible enzyme deacetylation. Nature. 2010;464(7285):121-125.

20. Zhao S, et al. Regulation of cellular metabolism by protein lysine acetylation. Science. 2010;327(5968):1000-1004.

21. Rardin MJ, et al. Label-free quantitative proteomics of the lysine acetylome in mitochondria identifies substrates of SIRT3 in metabolic pathways. Proc Natl Acad Sci USA. 2013;110(16):6601-6606.

22. Sankaralingam $\mathrm{S}$, et al. Lowering body weight in obese mice with diastolic heart failure improves cardiac insulin sensitivity and function: implications for the obesity paradox. Diabetes. 2015;64(5):1643-1657.

23. Scott I, Webster BR, Li JH, Sack MN. Identification of a molecular component of the mitochondrial acetyltransferase programme: a novel role for GCN5L1. Biochem J. 2012;443(3):655-661.

24. Russell LK, et al. Cardiac-specific induction of the transcriptional coactivator peroxisome proliferator-activated receptor gamma coactivator-1alpha promotes mitochondrial biogenesis and reversible cardiomyopathy in a developmental stage-dependent manner. Circ Res. 2004;94(4):525-533.

25. Jeninga EH, Schoonjans K, Auwerx J. Reversible acetylation of PGC-1: connecting energy sensors and effectors to guarantee metabolic flexibility. Oncogene. 2010;29(33):4617-4624.

26. Lopaschuk GD, Jaswal JS. Energy metabolic phenotype of the cardiomyocyte during development, differentiation, and postnatal maturation. J Cardiovasc Pharmacol. 2010;56(2):130-140.

27. Gomez-Arroyo J, et al. Metabolic gene remodeling and mitochondrial dysfunction in failing right ventricular hypertrophy secondary to pulmonary arterial hypertension. Circ Heart Fail. 2013;6(1):136-144.

28. Hsieh MC, Das D, Sambandam N, Zhang MQ, Nahlé Z. Regulation of the PDK4 isozyme by the Rb-E2F1 complex. J Biol Chem. 2008;283(41):27410-27417.

29. Piao L, et al. FOXO1-mediated upregulation of pyruvate dehydrogenase kinase-4 (PDK4) decreases glucose oxidation and impairs right ventricular function in pulmonary hypertension: therapeutic benefits of dichloroacetate. J Mol Med. 2013;91(3):333-346.

30. Zhang Y, et al. Estrogen-related receptors stimulate pyruvate dehydrogenase kinase isoform 4 gene expression. $J$ Biol Chem 2006;281(52):39897-39906.

31. Zhang L, Mori J, Wagg C, Lopaschuk GD. Activating cardiac E2F1 induces up-regulation of pyruvate dehydrogenase kinase 4 
in mice on a short term of high fat feeding. FEBS Lett. 2012;586(7):996-1003.

32. Santalucía T, et al. Developmental regulation of GLUT-1 (erythroid/Hep G2) and GLUT-4 (muscle/fat) glucose transporter expression in rat heart, skeletal muscle, and brown adipose tissue. Endocrinology. 1992;130(2):837-846.

33. Mori J, et al. Agonist-induced hypertrophy and diastolic dysfunction are associated with selective reduction in glucose oxidation: a metabolic contribution to heart failure with normal ejection fraction. Circ Heart Fail. 2012;5(4):493-503.

34. Horton JL, et al. Mitochondrial protein hyperacetylation in the failing heart. JCI Insight. 2016;2(1):e84897.

35. Lee CF, et al. Normalization of NAD+ Redox Balance as a Therapy for Heart Failure. Circulation. 2016;134(12):883-894.

36. Finck BN, et al. The cardiac phenotype induced by PPARalpha overexpression mimics that caused by diabetes mellitus. $J C l i n$ Invest. 2002;109(1):121-130.

37. Holland WL, et al. Inhibition of ceramide synthesis ameliorates glucocorticoid-, saturated-fat-, and obesity-induced insulin resistance. Cell Metab. 2007;5(3):167-179.

38. Stram AR, Wagner GR, Fogler BD, Pride PM, Hirschey MD, Payne RM. Progressive mitochondrial protein lysine acetylation and heart failure in a model of Friedreich's ataxia cardiomyopathy. PLoS One. 2017;12(5):e0178354.

39. Scott I, Webster BR, Chan CK, Okonkwo JU, Han K, Sack MN. GCN5-like protein 1 (GCN5L1) controls mitochondrial content through coordinated regulation of mitochondrial biogenesis and mitophagy. J Biol Chem. 2014;289(5):2864-2872.

40. Webster BR, Scott I, Traba J, Han K, Sack MN. Regulation of autophagy and mitophagy by nutrient availability and acetylation. Biochim Biophys Acta. 2014;1841(4):525-534.

41. Gao S, et al. Regulation of substrate oxidation preferences in muscle by the peptide hormone adropin. Diabetes. 2014;63(10):3242-3252.

42. Dominy JE, et al. The deacetylase Sirt6 activates the acetyltransferase GCN5 and suppresses hepatic gluconeogenesis. Mol Cell. 2012;48(6):900-913

43. Lopaschuk GD, Ussher JR, Folmes CD, Jaswal JS, Stanley WC. Myocardial fatty acid metabolism in health and disease. Physiol Rev. 2010;90(1):207-258.

44. Mori J, Alrob OA, Wagg CS, Harris RA, Lopaschuk GD, Oudit GY. ANG II causes insulin resistance and induces cardiac metabolic switch and inefficiency: a critical role of PDK4. Am J Physiol Heart Circ Physiol. 2013;304(8):H1103-H1113.

45. Wohlschlaeger J, et al. Reversible regulation of the retinoblastoma protein/E2F-1 pathway during "reverse cardiac remodelling" after ventricular unloading. J Heart Lung Transplant. 2010;29(1):117-124.

46. Nau PN, et al. Metabolic adaptation of the fetal and postnatal ovine heart: regulatory role of hypoxia-inducible factors and nuclear respiratory factor-1. Pediatr Res. 2002;52(2):269-278.

47. Choi YH, et al. Myocardial hypertrophy overrides the angiogenic response to hypoxia. PLoS One. 2008;3(12):e4042.

48. Kato T, et al. Analysis of metabolic remodeling in compensated left ventricular hypertrophy and heart failure. Circ Heart Fail. 2010;3(3):420-430.

49. Haycock GB, Schwartz GJ, Wisotsky DH. Geometric method for measuring body surface area: a height-weight formula validated in infants, children, and adults. J Pediatr. 1978;93(1):62-66.

50. Yatscoff MA, et al. Myocardial hypertrophy and the maturation of fatty acid oxidation in the newborn human heart. Pediatr Res. 2008;64(6):643-647.

51. Kampmann C, et al. Normal values of M mode echocardiographic measurements of more than 2000 healthy infants and children in central Europe. Heart. 2000;83(6):667-672.

52. Devore GR. The use of Z-scores in the analysis of fetal cardiac dimensions. Ultrasound Obstet Gynecol. 2005;26(6):596-598.

53. Lang RM, et al. Recommendations for chamber quantification. Eur J Echocardiogr. 2006;7(2):79-108.

54. Zhang L, et al. Cardiac insulin-resistance and decreased mitochondrial energy production precede the development of systolic heart failure after pressure-overload hypertrophy. Circ Heart Fail. 2013;6(5):1039-1048.

55. Morgunov I, Srere PA. Interaction between citrate synthase and malate dehydrogenase. Substrate channeling of oxaloacetate. J Biol Chem. 1998;273(45):29540-29544

56. Sambandam N, Steinmetz M, Chu A, Altarejos JY, Dyck JR, Lopaschuk GD. Malonyl-CoA decarboxylase (MCD) is differentially regulated in subcellular compartments by 5'AMP-activated protein kinase (AMPK). Studies using H9c2 cells overexpressing MCD and AMPK by adenoviral gene transfer technique. Eur J Biochem. 2004;271(13):2831-2840.

57. Branco AF, Pereira SP, Gonzalez S, Gusev O, Rizvanov AA, Oliveira PJ. Gene Expression Profiling of H9c2 Myoblast Differentiation towards a Cardiac-Like Phenotype. PLoS One. 2015;10(6):e0129303.

58. Fillmore N, et al. Effect of fatty acids on human bone marrow mesenchymal stem cell energy metabolism and survival. PLoS One. 2015;10(3): $\mathrm{e} 0120257$. 